\author{
ACTA MYCOLOGICA \\ Vol. 47 (2): 235-264 \\ 2012
}

\title{
Preliminary studies of fungi in the Biebrza National Park (NE Poland). II. Macromycetes
}

\author{
ANNA KUJAWA ${ }^{1}$, MARTA WRZOSEK ${ }^{2}$, GRAŻYNA DOMIAN ${ }^{3}$, KAMIL KEDRA $^{4}$, \\ JAROSŁAW SZKODZIK ${ }^{5}$, MARIA RUDAWSKA ${ }^{6}$, TOMASZ LESKI $^{6}$, LESZEK KARLINSKI ${ }^{6}$, \\ MARCIN PIETRAS ${ }^{6}$, BEAŻEJ GIERCZYK 7 , MARIA DYNOWSKA ${ }^{8}$, \\ DOMINIKA SLUSARCZYK ${ }^{9}$, IZABELA KAŁUCKA ${ }^{9}$ and MARIA ŁAWRYNOWICZ
}

\author{
${ }^{1}$ Institute for Agricultural and Forest Environment, Polish Academy of Sciences, Field Station in Turew; \\ Turew Szkolna 4, PL-64-000 Kościan, ankujawa@man.poznan.pl \\ ${ }^{2}$ Department of Plants Systematics and Geography, University of Warsaw \\ Al. Ujazdowskie 4, PL-00-478 Warszawa, mwrzosek@biol.uw.edu.pl \\ ${ }^{3}$ Regional Directorate for Environmental Protection in Szczecin \\ Jagiellońska 32, PL-70-382 Szczecin, grazyna.domian.szczecin@rdos.gov.pl \\ ${ }^{4}$ Polish Mycological Society, kamil_kedra@o2.pl \\ ${ }^{5}$ Nature \& Ecology of Łódź Macroregion Website, Ekolodzkie.pl, jaroslaw.szkodzik@ekolodzkie.pl \\ ${ }^{6}$ Laboratory of Mycorrhizal Research, Institute of Dendrology, Polish Academy of Sciences, \\ Parkowa 5, PL-62-035 Kórnik, mariarud@man.poznan.pl, tleski@man.poznan.pl, \\ leszekk@man.poznan.pl, mpietras@man.poznan.pl \\ ${ }^{7}$ Faculty of Chemistry, A. Mickiewicz University in Poznań, Grunwaldzka 6, PL-60-780 Poznań, \\ hanuman@amu.edu.pl \\ ${ }^{8}$ Department of Mycology, Warmia and Mazury University in Olsztyn \\ Oczapowskiego 1A, PL-10-957 Olsztyn, dynow@uwm.edu.pl \\ ${ }^{9}$ Department of Mycology, Faculty of Biology and Environmental Protection \\ University of Łódź, Banacha 12/16, PL-90-237 Łódź \\ dominika@biol.uni.lodz.pl, ikalucka@biol.uni.lodz.pl,miklaw@biol.uni.lodz.pl
}

Kujawa A., Wrzosek M., Domian G., Kędra K., Szkodzik J., Rudawska M., Leski T., Karliński L., Pietras M., Gierczyk B., Dynowska M., Ślusarczyk D., Kałucka I., Ławrynowicz M.: Preliminary studies of fungi in the Biebrza National Park (NE Poland). II. Macromycetes. Acta Mycol. 47 (2): 235-264, 2012.

This paper presents the second part of the results of the first short-term inventory of fungi species occurring in the Biebrza National Park and is focused on a survey of macrofungi. The total number of macrofungi found during the survey in late August 2012 was 289 taxa: 17 ascomycetes and 272 basidiomycetes, inhabiting diverse ecological niches. The identified fungi belonged to ectomycorrhizal symbionts and saprobionts decomposing wood, plant litter and animal remnants. The identity of 25 mycorrhizal fungi species has been confirmed using molecular techniques. Five species are newly reported for Poland (Amanita olivaceogrisea, Lepiota lepida, Stropharia alcis, Xerocomus fennicus, X. cisalpinus) and 225 taxa (79\%) were not known in the Biebrza National Park. Fifty nine species are endangered in Poland, of which nine species are protected by law. Data on species richness and the taxonomic diversity of the identified 
fungi are briefly commented in terms of their significance for nature conservation and future research.

Key words: all-species inventory, macrofungi, rare species, protected and threatened fungi, protected area

\section{INTRODUCTION}

This paper presents the second part of the results from a fungi survey carried out in the Biebrza National Park (BbNP) in early autumn 2012 within the framework of a scientific project by the Polish Mycological Society. This interdisciplinary research covered many diverse trophic, ecological and taxonomic groups of fungi. The text below is devoted to macromycetes. The role of macromycetes in estimating the state of the environment and in protection management is well documented and highly important. New data about macrofungi diversity in national parks will facilitate the inclusion of fungi in discussions on biodiversity and conservation decisions (Dahlberg et al. 2011).

National parks, as relatively large, natural (or close to natural) areas subjected to strict protection, are one of the best places for studies on fungal ecology and give a chance to distinguish species requiring special protection among those reacting negatively to the environmental changes caused by human activity. Furthermore, national parks, being a kind of enclave, provide conditions necessary for the survival of many species sensitive to the stress caused by human activity (anthropopressure).

Information about fungi in Polish national parks is incomplete. The majority of national parks have very limited data on mycobiota. In terms of mycological data, the Biebrza National Park has been left largely unexplored. The only relatively comprehensive research on fungi in the BbNP covered lichens (cf. Kolanko 2005; Dyrcz, Werpachowski 2005). Some data on macromycetes (Asco- and Basidiomycota) of the BbNP comes from a report on a mycological foray conducted in November 2010 within the framework of the XXXIII Wszechnica Biebrzańska Seminar. Sixty-six species of fungi collected during the foray, together with Bovista paludosa Lév. known from the literature from the 1960s, were listed by Kujawa et al. (2011). The later species, as well as five other macromycetes [i. e., Leccinum rotundifoliae (Singer) A.H. Sm., Thiers \& Watling, Suillus bovinus (Pers.) Rousse, Lactarius pubescens Fr., Hypholoma udum (Pers.) Quél. and Psilocybe turficola J. Favre] reported in the vicinity of Lipsk village (Anonymous 1968), cannot be strictly assigned to the area of the BbNP because the precise localities of the collecting sites have not been determined.

The basic information on the history of BbNP, its specific habitats and flora, as well as the background and the aims of the study are introduced in the first part of our report dealing with micromycetes (Ruszkiewicz-Michalska et al. 2012). The results of research on slime moulds, hypogeous fungi and Hebeloma spp. will be presented in separate papers at a later time. 


\section{MATERIALS AND METHODS}

Most details on the materials and methods used in the paper are presented by Ruszkiewicz-Michalska et al. (2012). In the present paper the macromycetes were determined in the same way that was accepted by Wojewoda (2003) and Chmiel (2006), The only exception is Onygena equina that, despite its small size, has been included in this paper. Macromycetes were collected in five protective units of the BbNP: Brzeziny, Grzędy, Kapice, Osowiec and Werykle and at the fortress in Osowiec-Twierdza (Fig. 1).

Standard methods of studying macrofungi, based on microscopic analyses, were used in the species identification (hand-made sections or squash preparations mounted in water, $3-5 \% \mathrm{KOH}, 10 \%$ ammonia, Congo Red [1\% solution of dye in $10 \%$ ammonia], Melzer's reagent; light microscope, magnification 100-1000x). Some of ectomycorrhizal fungi were identified using sequencing of the PCR amplified internal transcribed spacer (ITS) of n-rDNA based on protocols designated for the study of ectomycorrhizas (Leski et al. 2010; Rudawska et al. 2011).

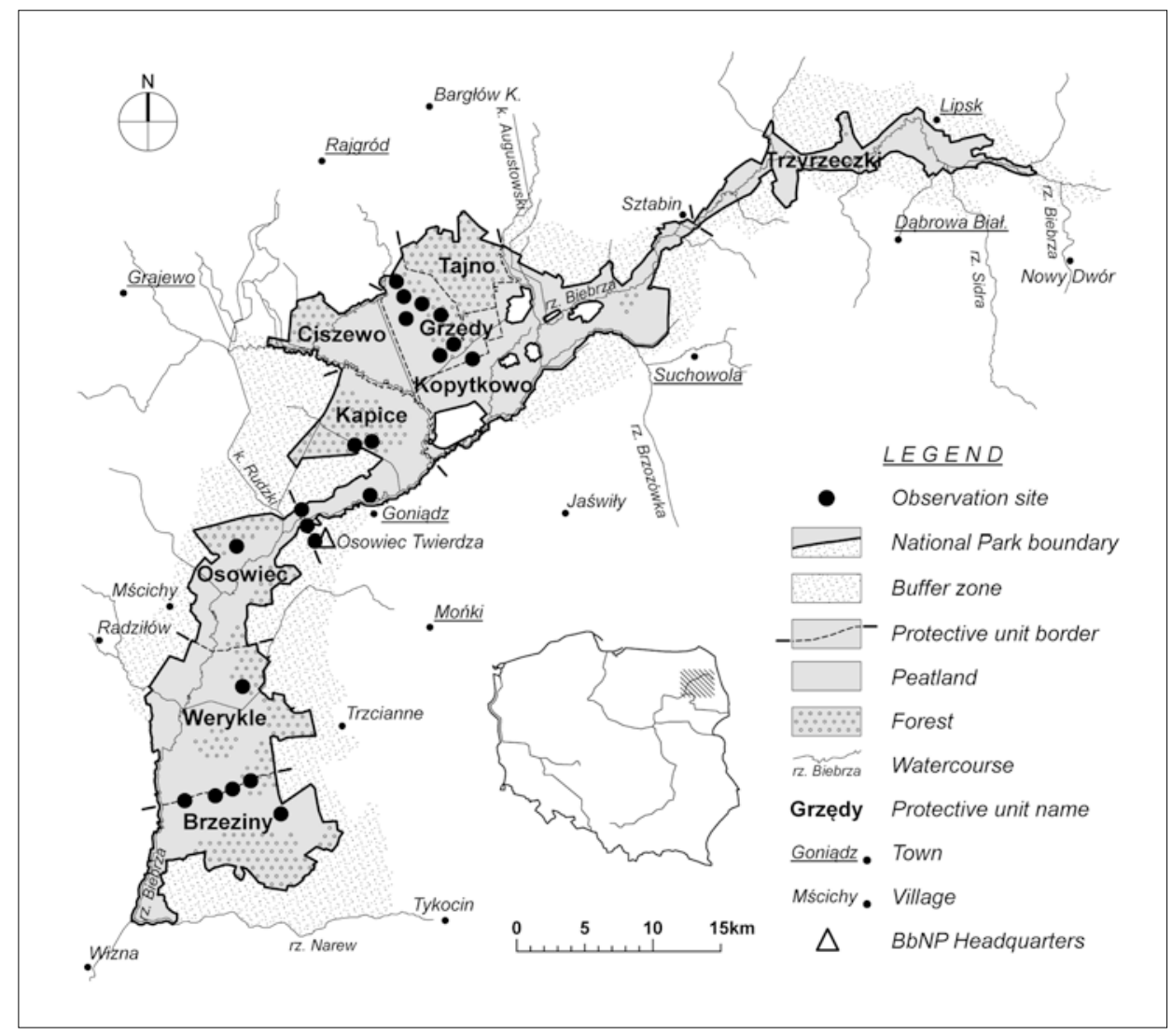

Fig. 1. Localization of observation sites in the Biebrza National Park (originally delineated by K. Kędra, based on materials provided by the BbPN office). 
The following monographs and keys were used in fungi identification: Bas et al. (1990, 1995, 1998, 1999), Knudsen, Vesterholt (2008) and Noordeloos et al. (2001, 2005). Taxonomic system of fungi of Kirk et al. (2008) is accepted while nomenclature follows Wojewoda (2003), Chmiel (2006), and Mułenko et al. (2008), and Index Fungorum (www.indexfungorum.org). Threat categories are listed according to Wojewoda and Ławrynowicz (2006), comments on the number of localities are based on Chmiel (2006), Wojewoda (2003) and Kujawa (2012) unless stated differently. Host plant species were determined using the key by Rutkowski (2004) and their names are given after Mirek et al. (2002). All photos are made by Grażyna Domian.

The specimens documenting research are deposited in the Herbarium of the Faculty of Biology, University of Warsaw (WA), Herbarium Universitatis Lodziensis (LOD) at the University of Lodz and in the Institute of Dendrology of the Polish Academy of Sciences in Kórnik.

\section{RESULTS}

In total, 289 taxa of macromycetes (species, varieties and forms) (17 Ascomycota and 272 Basidiomycota) belonging to 20 taxonomic groups were identified (Tab. 1). They include 25 taxa determined with the use of molecular methods (annotated in the species list). Trophic groups of fungi inhabiting diverse substrates include humicolous saprotrophs (e.g., Agrocybe erebia, Bovista spp., Lepiota spp.), saprotrophs on litter (e.g., Calocybe carnea, Coprinus lagopus, Crinipellis scabella, Mycena zephirus) and saprotrophs on wood (e.g., Bulgaria inquinans, Oligoporus ptychogaster, Paxillus atrotomentosus, Pluteus atricapillus), as well as plant parasites (e.g., Inonotus obliquus, Laetiporus sulphureus, Phellinus ignarius, Polyporus squamosus). Fungi fructificating on excrement form a separate group among the identified saprobionts (e.g., Coprotus luteus, Coprinus cinereus, C. miser, C. niveus, Panaeolus alcidis, Stropharia alcis). Single species growing on remnants of fungi (Asterophora lycoperdoides) and animals (Onygena eqiuna) have also been found. A number of interesting specimens still await determination.

Ectomycorrhizal fungi (e.g., Amanita spp., Laccaria spp., Lactarius spp., and Russula spp.) represent an important trophic group of fungi collected during the field

Table 1

Numbers of fungi taxa collected at the study area

\begin{tabular}{|c|c|c|c|c|c|c|c|c|c|c|c|c|c|c|c|c|c|c|c|c|c|}
\hline 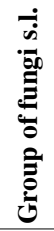 & 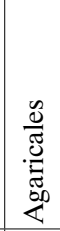 & 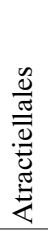 & 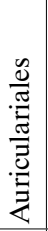 & $\begin{array}{l}\frac{\tilde{\theta}}{\tilde{J}} \\
\frac{\tilde{0}}{0} \\
0\end{array}$ & 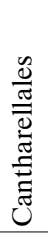 & 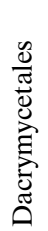 & 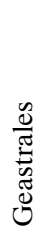 & 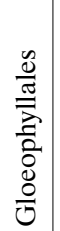 & $\begin{array}{l}\frac{0}{0} \\
\frac{0}{\pi} \\
\frac{0}{2} \\
0 \\
0 \\
0\end{array}$ & 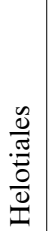 & 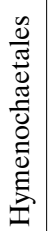 & 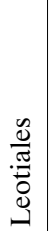 & 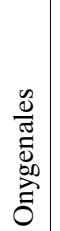 & 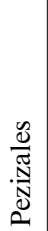 & $\begin{array}{l}\frac{\mathscr{E}}{\tilde{J}} \\
\frac{\vec{J}}{\tilde{\Xi}} \\
\frac{\pi}{2}\end{array}$ & 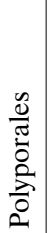 & 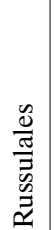 & $\begin{array}{l}\frac{0}{0} \\
\frac{\pi}{0} \\
\frac{0}{0} \\
\frac{0}{0} \\
\frac{D}{\sigma}\end{array}$ & 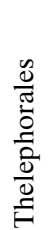 & 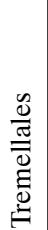 & 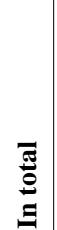 \\
\hline No. & \begin{tabular}{|l|l}
158 \\
\end{tabular} & 1 & 4 & 24 & 4 & 3 & 3 & 1 & 4 & 4 & 5 & 2 & 1 & 9 & 2 & 29 & 28 & 1 & 4 & 2 & 289 \\
\hline
\end{tabular}


survey in the BbNP. Most of them have been the fungal partner of Scots pine, oak, birch and hazel. The molecular methods proved to be a very useful tool in the identification of taxonomically difficult mycorrhizal fungal taxa (e.g., Russula spp.). Up to now species hardly distinguishable from closely related taxa have been rarely (e.g., Cortinarius illibatus) or never recorded in Poland (Xerocomus cisalpinus, X. fennicus).

The following abbreviations are used in the list of species: BPU - Brzeziny protective unit (see Fig. 1); KPU - Kapice protective unit; GPU - Grzędy protective unit; OPU - Osowiec protective unit; WPU - Werykle protective unit; T-C - Tilio cordatae-Carpinetum betuli; V-P - Vaccinio uliginosi-Pinetum. Initials of the names are given to denote collecting and/or identifying person (e.g., GD - Grażyna Domian); * - taxa new for Poland; Ex, E, V, R, I - IUCN category; $\mathbf{P}$ - protected species.

\section{ASCOMYCOTA}

Bulgaria inquinans (Pers.) Fr. on log of Carpinus betulus L., GPU, T-C, 29 Aug., leg. $\&$ det. GD; on log of Quercus robur L., GPU, T-C, 29 Aug., vid. GD.

Chlorociboria aeruginascens (Nyl.) Kanouse ex C.S. Ramamurthi, Korf \& L.R. Batra on wood debris, KPU, T-C, 28 Aug., leg. \& det. $M W$; on wood of deciduous trees, GPU, mixed forest, 29 Aug., leg. AK\& ES, det. AK; on log of Populus sp., GPU, T-C, 29 Aug., leg. \& det. GD;on on wood of deciduous trees, GPU, thickets, 30 Aug., leg. $\&$ det. $M W$; on wood of Betula pendula Roth, WPU, Barwik, coniferous forest, 31 Aug., leg. \& det. $M W$.

Coprotus luteus Kimbr. on elk (?) excrement, GPU, mixed forest, 29 Aug., leg. GD, det. $A K$.

Note. Species known in Poland from a single locality in Wigierski National Park (Halama, Romański 2010).

Helvella crispa (Scop.) Fr. on ground, GPU, deciduous forest, 29 Aug., leg. \& det. AK; on ground, GPU, thickets, 30 Aug., leg. $J S \& G D$, det. $J S \& G D$.

Helvella lacunosa Afzel. on ground, GPU, roadside, 29 Aug., leg. \& det. GD; R.

Helvella macropus (Pers.) P. Karst. on ground, KPU, T-C, 28 Aug., leg. \& det. DŚ; on ground, GPU, deciduous forest, 29 Aug., leg. Magdalena Maliszewska \& AK, det. AK.

Humaria hemisphaerica (F.H. Wigg.) Fuckel on ground, KPU, T-C, 28 Aug., leg. \& det. $M \longleftarrow$.

Hymenoscyphus albidus (Roberge ex Desm.) W. Phillips s.l. on petioles of Fraxinus excelsior L. leaves, GPU, deciduous forest, 29 Aug., leg. \& det. AK.

Hymenoscyphus calyculus (Sowerby) W. Phillips on wood debris, KPU, T-C, 28 Aug., leg. $M W, \operatorname{det} . A K$.

Hymenoscyphus fructigenus (Bull.) Gray on acorns, KPU, T-C, 28 Aug., leg. AK, DŚ \& $J S$, det. AK; on acorns, GPU, deciduous forest, 29 Aug., leg. \& det. AK.

Leotia lubrica (Scop.) Pers. on ground, GPU, T-C, 29 Aug., leg. \& det. GD.

Onygena equina (Willdenow) Pers. ex Fr. on hoof of elk(?), OPU, Sośnia, coniferous forest, 01 Sept., leg. \& det. GD; on hoof of elk(?), GPU, coniferous forest, 29 Aug., leg. \& det. GD; R. (Fig. 2)

Note. Species recorded in Poland in tens localities (Mułenko et al. 2008; Kujawa 2012). 
Otidea concinna (Pers.) Sacc. on ground, KPU, deciduous forest, 28 Aug., leg. \& det. $M R, T L, M P \& L K$ (molecular data).

Nоте. Species recorded in Poland in a few localities.

Otidea onotica (Pers.) Fuckel on ground, KPU, T-C, 28 Aug., leg. MD \& JS, det. MD; on ground, GPU, mixed forest, 29 Aug., leg. \& det. $M W$.

Pyronema domesticum (Sowerby) Sacc. on post fire place, WPU, Barwik, lawn at the recreational place, 31 Aug., leg. \& det. $A K$.

Note. Species known in Poland from a single locality (Mleczko 2004).

Scutellinia scutellata (L.) Lambotte on wood debris, GPU, birch forest, 30 Aug., leg. $G D, \operatorname{det}$. $A K$.

Tarzetta cupularis (L.) Svrček on ground, KPU, T-C, 28 Aug., leg. MW, AK\&MŁ, det. AK.

\section{BASIDIOMYCOTA}

Agrocybe erebia (Fr.) Kühner ex Singer on ground, KPU, T-C, 28 Aug., leg. AK, det. $B G$.

Amanita argentea Huijsman on ground, GPU, thickets, 30 Aug., leg. $A K, M W, K K$, $G D, M R, T L, L K \& M P$, det. $A K, M R, T L, L K \& M P$ (molecular data).

Note. Species known in Poland from a single locality (Halama 2009).

Amanita citrina (Schaeff.) Pers. on ground, KPU, T-C, 28 Aug., leg. \& det. MW; on ground, GPU, thickets, 30 Aug., leg. \& det. $M W$.

Amanita fulva (Schaeff.) Pers. on ground, GPU, deciduous forest, 29 Aug., leg. \& det. $A K$; on ground, GPU, thickets, 30 Aug., leg. \& det. $M W$; on ground, WPU, Barwik, coniferous forest, 31 Aug., leg. \& det. $M W$.

Amanita muscaria (L.) Hook. on ground, KPU, T-C, 28 Aug., leg. \& det. TL; on ground, WPU, Barwik, coniferous forest, 31 Aug., leg. \& det. MR.

*Amanita olivaceogrisea Kalamees on ground, KPU, deciduous forest, 28 Aug., leg. \& det. $M R, T L, M P \& L K$ (molecular data).

Note. Species hitherto not reported in Polish literature, known also from Kampinos National Park (Kujawa, unpubl. data).

Amanita pantherina (DC.) Krombh. on ground, KPU, T-C, 28 Aug., leg. TL, det. IK (molecular data); on ground, GPU, thickets, 30 Aug., leg. \& det. $M W \& K K$.

Amanita phalloides (Vaill.) Link on ground, KPU, T-C, 28 Aug., leg. \& det. TL (molecular data); on ground, GPU, T-C, 29 Aug., vid. GD; on ground, GPU, thickets, 30 Aug., leg. \& det. AK.

Amanita porphyria (Alb. \& Schwein.) Mladý on ground, GPU, mixed forest, 29 Aug., leg. $K K$, det. $A K$; on ground, GPU, thickets, 30 Aug., leg. \& det. JS; on ground, GPU, $T-C, 30$ Aug., leg. GD, det. $B G$.

Amanita rubescens (Pers.) Gray on ground, KPU, T-C, 28 Aug., leg. \& det. GD, AK; on ground, GPU, thickets, 30 Aug., leg. \& det. $M W$; on ground, WPU, Barwik, coniferous forest, 31 Aug., leg. \& det. AK. 
Amanita vaginata (Bull.) Vittad. on ground, GPU, mixed forest, 30 Aug., leg. \& det. $M R, T L, M P \& L K$ (molecular data); on ground, WPU, Barwik, coniferous forest, 31 Aug., leg. GD, det. $A K$.

Antrodiella serpula (P. Karst.) Spirin \& Niemelä [ = A. hoehnelii (Bres.) Niemelä] on $\log$ of Alnus glutinosa (L.) Gaertn., GPU, T-C, 29 Aug., leg. \& det. GD; R.

Armillaria borealis Marxm. \& Korhonen on wood, KPU, T-C, 28 Aug., leg. \& det. $A K$; on wood of deciduous trees, GPU, deciduous forest, 29 Aug., leg. \& det. AK; on ground, GPU, deciduous forest, 30 Aug., vid. $M W$; on ground, WPU, Barwik, thickets, 31 Aug., leg. \& det. $M W$.

Asterophora lycoperdoides (Bull.) Ditmar on Lactarius?, GPU, T-C, 29 Aug., leg. \& det. $G D ; \mathbf{R}$.

Auricularia auricula-judae (Bull.) Wettst. on wood, Osowiec-Twierdza, roadside, 31 Aug., leg. \& det. $G D \& K K$.

Auriscalpium vulgare Gray on cone of Pinus, KPU, T-C, 28 Aug., leg. \& det. MD; on cone of Pinus sylvestris L., GPU, mixed forest, 29 Aug., leg. \& det. MW.

Baeospora myosura (Fr.) Singer on cone of Picea abies (L.) H. Karst., GPU, T-C, 30 Aug., leg. \& det. GD.

Bjerkandera adusta (Willd.) P. Karst. on wood of deciduous tree, KPU, T-C, 28 Aug., leg. $M W$, det. $M W \& J S$; on wood of deciduous trees, GPU, mixed forest, 29 Aug., leg. $\&$ det. MW; on Populus tremula L., WPU, Barwik, coniferous forest, 31 Aug., leg. \& det. GD.

Bolbitius titubans (Bull.) Fr. on ground, GPU, mixed forest, 29 Aug., leg. GD, det. AK; on plant debris, WPU, Barwik, roadside, 31 Aug., leg. MR, det. AK.

Boletus edulis Bull. on ground, GPU, mixed forest, 29 Aug., leg. \& det. KK; on ground, GPU, thickets, 30 Aug., leg. \& det. MW.

Bovista dermoxantha (Vittad.) De Toni [ = B. pusilla (Batsch) Pers.] on ground, GPU, dune, 30 Aug., leg. $J S, M W, A K, G D \& K K$, det. $M W$; on ground, WPU, Barwik, roadside, 31 Aug., leg. GD, det. $A K$.

Bovista nigrescens Pers. on ground, KPU, T-C, 28 Aug., leg. \& det. AK.

Bovista plumbea Pers. on ground, Osowiec-Twierdza, area of the fortress, 31 Aug., leg. $M W$, det. $A K$.

Calocera cornea (Batsch) Fr. on wood of deciduous trees, GPU, mixed forest, 29 Aug., leg. \& det. $M W$.

Calocera furcata (Batsch) Fr. on wood, WPU, Barwik, coniferous forest, 31 Aug., leg. $M W, \operatorname{det} . A K ; \mathbf{R}$.

Calocera viscosa (Pers.) Fr. on wood of coniferous trees, GPU, deciduous forest, 29 Aug., leg. Magdalena Maliszewska, det. AK.

Calocybe carnea (Bull.) Donk on litter, KPU, T-C, 28 Aug., leg. \& det. AK.

Calvatia excipuliformis (Scop.) Perdeck on ground, WPU, Barwik, coniferous forest 31 Aug., leg. GD, det. $A K$. 
Calvatia utriformis (Bull.) Jaap on ground, KPU, T-C, 28 Aug., leg. \& det. MD, AK; on Salix sp., GPU, thickets, 30 Aug., leg. \& det. AK; on ground, GPU, dune, 30 Aug., leg. \& det. $M W \& K K$.

Cantharellula umbonata (J.F. Gmel.) Singer on ground, WPU, Barwik, coniferous forest, 31 Aug., leg. $M W$, det. $A K$.

Cantharellus cibarius Fr. on ground, KPU, T-C, 28 Aug., leg. \& det. TL, MW; on ground, GPU, deciduous forest, 29 Aug., leg. \& det. $A K$; on ground, GPU, thickets, 30 Aug., leg. \& det. $M W$.

Chroogomphus rutilus (Schaeff.) O.K. Mill. on ground, GPU, thickets, 30 Aug., leg. $G D$, det. JS.

Clavicorona pyxidata (Pers.) Doty on wood of Corylus avellana L., GPU, mixed forest, 29 Aug., leg. \& det. MŁ; on log of Betula pendula Roth, GPU, T-C, 30 Aug., vid. $G D$; on wood, Osowiec-Twierdza, area of the fortress, 31 Aug., leg. \& det. $M W ; \mathbf{V}$.

Clavulina cinerea (Bull.) J. Schröt. on ground, KPU, T-C, 28 Aug., leg. \& det. IK; on ground, GPU, near Góra Solistowska, mixed forest, 29 Aug., leg. GD, det. BG.

Clavulina coralloides (L.) J. Schröt. on ground, KPU, educational path "Brzeziny Kapickie", birch forest, 29 Aug., leg. Magdalena Maliszewska, det. AK; on ground, GPU, mixed forest, 29 Aug., leg. IK, det. IK\& $A K$; on ground, GPU, mixed forest, 29 Aug., leg. \& det. $M W$; on ground, GPU, T-C, 29 Aug., leg. \& det. GD.

Climacocystis borealis (Fr.) Kotl. \& Pouzar at the base of Picea abies (L.) H. Karst., GPU, T-C, 29 Aug., leg. \& det. GD; at the base of Picea abies (L.) H. Karst., GPU, $T-C, 30$ Aug., leg. \& det. GD; R.

Clitocybe clavipes (Pers.) P. Kumm. on ground, KPU, T-C, 28 Aug., leg. \& det. IK; on ground, GPU, mixed forest, 29 Aug., leg. \& det. $M W$; on ground, GPU, thickets, 30 Aug., leg. \& det. GD \& JS; on ground, WPU, Barwik, coniferous forest, 31 Aug., leg. $\&$ det. $K K, M W \& A K$.

Clitocybe gibba (Pers.) P. Kumm. on ground, KPU, T-C, 28 Aug., leg. $A K \&$ GD, det. $A K$; on ground, GPU, deciduous forest, 29 Aug., leg. $K K$, det. $A K$; on ground, GPU, thickets, 30 Aug., leg. $G D$, det. $A K$; on ground, WPU, Barwik, coniferous forest, 31 Aug., leg. \& det. $K K, M W \& A K$.

Clitocybe hydrogramma (Bull.) P. Kumm. on ground, GPU, mixed forest, 29 Aug., leg. $\&$ det. $A K \& M t ; \mathbf{R}$.

Clitocybe odora (Bull.) P. Kumm. on ground, GPU, deciduous forest, 29 Aug., leg. \& det. $A K$.

Clitopilus hobsoni (Berk. \& Broome) P.D. Orton on wood of deciduous trees, GPU, T-C, 29 Aug., leg. \& det. $A K$; on beaverboard, GPU, hunting pulpit, 30 Aug., leg. $G D$, det. $B G$; on Populus tremula L., WPU, Barwik, coniferous forest, 31 Aug., leg. GD, det. AK.

Collybia tuberosa (Bull.) P. Kumm. on debris of fungi, KPU, T-C, 28 Aug., leg. DŚ, det. ME. Coltricia perennis (L.) Murrill on ground, BPU, near Grobla Honczarowska, 01 Sept., coniferous forest, leg. \& det. GD.

Conocybe albipes (G.H. Otth.) Hauskn. var. albipes, on ground, WPU, educational path Biały Grąd, 01 Sept., pasture, leg. $G D \& K K$, det. $B G$. 
Conocybe apala (Fr.) Arnolds on ground, GPU, deciduous forest, 29 Aug., leg. AK, det. $B G$.

Note. Species known in Poland from two localities (Wojewoda 2003; Halama, Romański 2010).

Conocybe mesospora Kühner ex Watling on ground, KPU, T-C, 28 Aug., leg. AK, det. BG. Conocybe microspora (Velen.) Dennis var. microspora on ground, KPU, T-C, 28 Aug., leg. $A K$, det. $B G$.

Note. Species known in Poland from a single locality (Bujakiewicz 2011).

Conocybe semiglobata Kuhner \& Watling var. semiglobata on ground, KPU, T-C, 28 Aug., leg. $A K$, det. $B G$.

Coprinus atramentarius (Bull.) Fr. on ground, KPU, T-C, 28 Aug., leg. $A K$, det. $B G$; on wood, GPU, T-C, 29 Aug., leg. GD, det. BG.

Coprinus cinereus (Schaeff.) Gray, on horse excrement, GPU, T-C, 29 Aug., leg. GD, det. $B G$.

Coprinus comatus (O.F. Mull.) Pers. on ground, GPU, T-C, 29 Aug., leg. \& det. GD.

Coprinus disseminatus (Pers.) Gray on stumps of deciduous trees, GPU, deciduous forest, 29 Aug., leg. \& det. AK; at the base of Populus sp., GPU, T-C, 30 Aug., leg. \& det. GD; at the base on Acer negundo L., Osowiec-Twierdza, area of the fortress, 31 Aug., leg. \& det. $M W$.

Coprinus domesticus (Bolton) Gray on wood, Goniądz, riverside, 31 Aug., leg. GD, det. $B G$.

Coprinus ellisii P.D.Orton on ground, GPU, near Góra Solistowska, T-C, 29 Aug., leg. $G D$, det. $B G$.

Note. Species known in Poland from two localities (Gierczyk et al. 2011b).

Coprinus jonesii Peck on ground, GPU, Ur. Dęby, roadside, 29 Aug., leg. GD, det. $B G$.

Note. Species known in Poland from few localities (Gierczyk et al. 2011b).

Coprinus lagopus (Fr.) Fr. var. lagopus on litter, WPU, educational path Biały Grąd, 01 Sept., pasture, leg. $G D$, det. $B G$.

Coprinus leiocephalus P.D.Orton on ground, GPU, deciduous forest, 29 Aug., leg. AK, det. $B G$.

Coprinus micaceus (Bull.) Fr. on ground, GPU, roadside, under Picea abies (L.) H. Karst., 29 Aug., leg. $A K$, det. $B G$; on wood, GPU, T-C, 30 Aug., leg. \& det. GD; on wood, WPU, Barwik, mixed forest, 31 Aug., leg. $G D \& A K$, det. $B G$.

Coprinus miser P. Karst. on horse excrement, GPU, T-C, 29 Aug., leg. GD, det. BG.

Note. Species known in Poland from five localities (Gierczyk et al. 2011b).

Coprinus niveus (Pers.) Fr. on horse excrement, GPU, T-C, 29 Aug., leg. GD, det. BG; R. Coprinus plicatilis (Curtis) Fr. on ground, Osowiec-Twierdza, lawn, 31 Aug., leg. GD, det. $B G$;

Coprinus romagnesianus Singer on ground, GPU, roadside, 29 Aug., leg. GD, det. BG. Note. Species known in Poland from two localities (Gierczyk et al. 2011b). 
Coprinus xanthothrix Romagn. on litter, Osowiec-Twierdza, lawn, 28 Aug., leg. GD, det. $B G$; on litter, KPU, $T-C, 28$ Aug., leg. $A K$, det. $B G$; on wood, GPU, deciduous forest, 29 Aug., leg. $A K$, det. BG; on litter, WPU, Barwik, mixed forest, 31 Aug., leg. $G D$, det. $B G$.

Cortinarius armillatus (Fr.) Fr. on ground, BPU, Grobla Honczarowska, thickets, 01 Sept., leg. $K K \& G D$, det. $A K$.

Cortinarius cinnamomeus (L.) Fr. on ground, WPU, Barwik, coniferous forest, 31 Aug., leg. \& det. $M W$.

Cortinarius illibatus Fr. on ground, KPU, deciduous forest, 28 Aug., leg. \& det. MR, $T L, M P \& L K$ (molecular data); on ground, GPU, mixed forest, 30 Aug., leg. \& det. $M R, T L, M P \& L K$ (molecular data).

Note. Species known in Poland from one locality (Ronikier 2012).

Cortinarius violaceus (L.) Gray on ground, BPU, Grobla Honczarowska, scrubs, 01 Sept., leg. \& det. KK\& GD; V.

Crepidotus crocophyllus (Berk.) Sacc. on log of Populus tremula L., GPU, near Góra Solistowska, T-C, 29 Aug., leg. GD, det. BG.

Notes. Species recorded in Poland only in Brzeziczno Reserve (Flisińska 2004) and Białowieża Forest (Karasiński et al 2009; Bujakiewicz, Kujawa 2010).

Crepidotus mollis (Schaeff.) Staude var. calolepis (Fr.) Pilát on wood of deciduous trees, GPU, deciduous forest, 29 Aug., leg. \& det. AK; on wood, WPU, Barwik, coniferous forest, 31 Aug., leg. \& det. $M W$.

Note. The variety recorded in Poland in few localities.

Crepidotus mollis var. mollis on wood of deciduous trees, GPU, deciduous forest, 29 Aug., leg. \& det. $A K$; on wood, WPU, Barwik, coniferous forest, 31 Aug., leg. $M W$, det. $A K$.

Crepidotus variabilis (Pers.) P. Kumm. on wood of deciduous trees, GPU, deciduous forest, 30 Aug., leg. JS, det. AK.

Crinipellis scabella (Alb. \& Schwein.) Murrill on litter, GPU, educational path Wydmy, dune, 30 Aug., leg. GD, det. $A K$.

Cyathus striatus (Huds.) Willd. on wood, GPU, T-C, 13 Sept., vid. GD.

Cystoderma amianthinum (Scop.) Fayod on ground, GPU, T-C, 29 Aug., leg. GD, det. $B G$; on ground, GPU, mixed forest, 29 Aug., leg. $G D$, det. $B G$; on ground, GPU, Czerwone Bagno, roadside, 29 Aug., leg. GD, det. BG; on ground, WPU, Barwik, coniferous forest, 31 Aug., leg. KK, det. AK.

Cystodermella granulosum (Batsch) Harmaja on ground, WPU, Barwik, coniferous forest, 31 Aug., leg. $K K$, det. $A K$; I.

Cystolepiota seminuda (Lasch) Bon on ground, GPU, deciduous forest, 29 Aug., leg. \& det. $A K$; on ground, GPU, roadside, 29 Aug., leg. $G D$, det. $B G$; on ground, GPU, Czerwone Bagno, T-C, 29 Aug., leg. GD, det. BG.

Daedaleopsis confragosa (Bolton) J. Schröt. on wood of Betula, KPU, T-C, 28 Aug., leg. $G D \& A K$, det. $G D$; on wood of deciduous trees, KPU, educational path "Brzeziny Kapickie”, birch forest, 29 Aug., leg. Magdalena Maliszewska, det. AK. 
Delicatula integrella (Pers.) Fayod on stump of deciduous tree, KPU, T-C, 28 Aug., leg. \& det. AK; on wood of deciduous trees, GPU, deciduous forest, 29 Aug., leg. \& det. $A K$.

Disciseda bovista (Klotzsch) Henn. on ground, Osowiec-Twierdza, roadside, 31 Aug., leg. $G D \& K K$, det. $A K ; \mathbf{E}$.

Entoloma undatum (Gillet) M.M. Moser on ground, KPU, T-C, 28 Aug., leg. \& det. AK.

Exidia glandulosa (Bull.) Fr. on wood of Quercus robur L., KPU, T-C, 28 Aug., leg. \& det. $A K \& M W ; \mathbf{R}$.

Exidia plana (Wigg.) Donk on wood of Betula pendula Roth, GPU, mixed forest, 29 Aug., leg. \& det. JS.

Fistulina hepatica (Schaeff.) With. at base of Quercus robur L., GPU, T-C, 13 Sept., vid. $G D ; \mathbf{P}, \mathbf{R}$.

Fomes fomentarius (L.) J. Kickx f. on log of Betula, KPU, T-C, 28 Aug., leg. \& det. MW; on wood of Betula pendula Roth, GPU, deciduous forest, 29 Aug., leg. \& det. MW.

Fomitopsis pinicola (Sw.) P. Karst. on log of Betula pendula Roth, KPU, T-C, 28 Aug., leg. $M W \& A K$, det. $A K$; on wood of Picea abies (L.) H. Karst., GPU, mixed forest, 29 Aug., leg. \& det. JS.

Ganoderma applanatum (Pers.) Pat. on wood of deciduous trees, GPU, deciduous forest, 29 Aug., leg. \& det. AK; on wood, WPU, Barwik, coniferous forest, 31 Aug., leg. \& det. $M W$.

Ganoderma lucidum (Curtis) P. Karst. on log of Quercus robur L., GPU, T-C, 07 Sept., vid. GD; on log of Betula pendula Roth, GPU, Uroczysko Dęby, T-C, 12 Sept., vid. GD; on Quercus robur L., GPU, T-C, 15 Sept., vid. GD; P, R. (Fig. 3)

Geastrum fimbriatum Fr. on ground, OPU, near Sośnia, dune, 31 Aug., leg. Cezary Werpachowski, det. AK; $\mathbf{P}, \mathbf{R}$.

Geastrum minimum Schwein. on ground, OPU, near Sośnia, dune,, 31 Aug., leg. Cezary Werpachowski, det. AK; $\mathbf{P , \mathbf { E } .}$

Geastrum rufescens Pers. on ground, GPU, T-C, 29 Aug., leg. \& det. GD; P, E.

Gloeophyllum odoratum (Wulfen) Imazeki on woood of Picea abies (L.) H. Karst., GPU, T-C, 29 Aug., leg. \& det. GD.

Gloeoporus dichrous (Fr.) Bres. on wood of deciduous trees, GPU, deciduous forest, 29 Aug., leg. \& det. AK; E.

Gloeoporus taxicola (Pers.) Gilb. \& Ryvarden on log of Pinus sylvestris L., GPU, V-P, 29 Aug., leg. \& det. GD; R.

Gomphidius glutinosus (Schaeff.) Fr. on ground, WPU, Barwik, coniferous forest, 31 Aug., leg. MR, det. TL; R.

Gymnopilus penetrans (Fr.) Murrill on wood of deciduous trees, GPU, deciduous forest, 29 Aug., leg. \& det. MW; on ground, WPU, Barwik, coniferous forest, 31 Aug., leg. $G D$, det. $A K$.

Gymnopilus sapineus (Fr.) Maire on wood of deciduous trees, GPU, deciduous forest, 29 Aug., leg. \& det. AK. 
Gymnopus confluens (Pers.) Antonín, Halling \& Noordel. on litter, KPU, T-C, 28 Aug., leg. \& det. AK; on litter, GPU, deciduous forest, 29 Aug., leg. \& det. AK.

Gymnopus dryophilus (Bull.) Murrill on litter, KPU, T-C, 28 Aug., leg. \& det. MW.

Gymnopus peronatus (Bolton) Antonín, Halling \& Noordel. on ground, KPU, T-C, 28 Aug., leg. $M W, J S \& K K$, det. $M W$; on ground, GPU, deciduous forest, 29 Aug., leg. \& det. $A K$; on ground, GPU, thickets, 30 Aug., leg. \& det. $A K$.

Gyroporus castaneus (Bull.) Quél. on ground, GPU, coniferous forest, 30 Aug., leg. $\&$ det. $G D ; \mathbf{R}$.

Gyroporus cyanescens (Bull.) Quél. on ground, GPU, deciduous forest, 29 Aug., leg. $\&$ det. IK\& KK; $\mathbf{R}$.

Hapalopilus nidulans (Fr.) P.Karst. on wood of Betula pendula Roth, GPU, deciduous forest, 29 Aug., leg. \& det. MŁ \& JS.

Heterobasidion annosum (Fr.) Bref. s.l. on stump of deciduous trees, GPU, deciduous forest, 29 Aug., leg. \& det. MŁ \& IK.

Hydnellum concrescens (Pers.) Banker on ground, BPU, Grobla Honczarowska, thickets, 01 Sept., leg. $G D \& K K$, det. $B G$; P, E. (Fig. 4)

Hydnum rufescens Fr. on ground, GPU, mixed forest, 29 Aug., leg. \& det. AK.

Hygrocybe conica (Schaeff.) P. Kumm. var. conicopalustris (R. Haller Aar.) Heinem. on ground among Sphagnum sp., WPU, Barwik, peatbog, 31 Aug., leg. GD, det. AK.

Notes. Variety is often not distinguished from typical variety. Occurs in moist habitats. Distribution in Poland is unknown.

Hygrophoropsis aurantiaca (Wulfen) J. Schröt. on ground, GPU, mixed forest, 29 Aug., leg. \& det. $M W$; on ground, WPU, Barwik, coniferous forest, 31 Aug., leg. \& det. MW.

Hymenochaete rubiginosa (Schrad.) Lév. on stump of Quercus robur L., KPU, T-C, 28 Aug., leg. \& det. MW; on wood of Quercus robur L., GPU, deciduous forest, 29 Aug., leg. \& det. $A K \& J S$.

Hypholoma capnoides (Fr.) P. Kumm. on wood, WPU, Barwik, mixed forest, 31 Aug., leg. GD, det. $A K$.

Hypholoma fasciculare (Huds.) P. Kumm. var. pusillum J.E. Lange on wood, GPU, mixed forest, 30 Aug., leg. $G D$, det. $B G$.

NoTEs. Variety is often not distinguished from typical variety. Distribution in Poland is unknown.

Hypholoma sublateritium (Fr.) Quél. on wood, GPU, T-C, 30 Aug., leg. GD, det. BG.

Hypholoma subviride (Berk. \& M.A. Curtis) Dennis on stump and log of deciduous tree, KPU, T-C, 28 Aug., leg. $M W$, det. $A K$; on wood of deciduous trees, GPU, deciduous forest, 29 Aug., leg. $M D$, det. $A K$.

Inocybe appendiculata Kühner on ground, GPU, T-C, 30 Aug., leg. GD, det. BG.

Noте. Species known in Poland from a two locality in Tatry Mountains (Wojewoda 2003).

Inocybe asterospora Quél. on ground, GPU, thickets, 30 Aug., leg. \& det. MW.

Inocybe geophylla (Fr.) P. Kumm. var. geophylla on ground, KPU, T-C, 28 Aug., leg. $\&$ det. AK; on ground, GPU, mixed forest, 29 Aug., leg. \& det. KK; on ground, GPU, thickets, 30 Aug., leg. \& det. $M W$. 
Inonotus obliquus (Pers.) Pilát on Betula pendula Roth, GPU, V-P, 29 Aug., leg. \& det. GD; on Betula pendula Roth, GPU, near Góra Solistowska, thickets, 29 Aug., vid. GD; on Betula pendula Roth, GPU, deciduous forest, 30 Aug., leg. \& det. MW; P, R.

Irpex (Steccherinum) ochraceus (Pers.) Kotir. \& Saaren. on wood of deciduous tree, KPU, $T-C, 28$ Aug., leg. \& det. $M \longleftarrow \& J S$; on wood of deciduous trees, GPU, mixed forest, 29 Aug., leg. \& det. JS.

Ischnoderma benzoinum (Wahlenb.) P. Karst. on wood of Picea abies (L.) H. Karst., GPU, T-C, 30 Aug., leg. \& det. GD; V.

Laccaria amethystina (Bull.) Murrill on ground, GPU, deciduous forest, 29 Aug., leg. \& det. MŁ.

Laccaria bicolor (Maire) P.D. Orton on ground, WPU, Barwik, coniferous forest, 31 Aug., leg. \& det. $M W$.

Laccaria laccata (Scop.) Berk. \& Broome on ground, KPU, T-C, 28 Aug., leg. \& det. $A K$; on ground, KPU, educational path "Brzeziny Kapickie", birch forest, 29 Aug., leg. Magdalena Maliszewska, det. AK; on ground, GPU, mixed forest, 29 Aug., leg. \& det. $M R, T L, M P \& L K$ (molecular data); on ground, GPU, birch forest, 30 Aug., leg. $M W$, det. $A K$.

Laccaria laccata var. pallidifolia (Peck) Peck on ground, KPU, T-C, 28 Aug., leg. $M W$, det. $A K$; on ground, O.O. Grzędy, birch forest, 30 Aug., leg. \& det. $M R, T L, L K \& M P$. Notes. Variety is often not distinguished from typical variety. Distribution in Poland is unknown.

Laccaria proxima (Boud.) Pat. on ground, GPU, V-P, 29 Aug., leg. \& det. AK; on ground, GPU, birch forest, 30 Aug., leg. GD, det. $A K$.

Laccaria tortilis (Bolton) Cooke on ground, WPU, Barwik, coniferous forest, 31 Aug., leg. MR, det. TL.

Lactarius camphoratus Fr. on ground, GPU, deciduous forest, 29 Aug., leg. \& det. IK $\& A K$.

Lactarius glyciosmus (Fr.) Fr. on ground, KPU, T-C, 28 Aug., leg. \& det. AK; on ground, GPU, mixed forest, 29 Aug., leg. \& det. $M W$.

Lactarius helvus (Fr.) Fr. on ground, GPU, mixed forest, 30 Aug., leg. \& det. MR, TL, $M P \& L K$ (molecular data).

Lactarius necator (J.F. Gmel.) Pers. on ground, KPU, T-C, 28 Aug., leg. MW, det. $A K$; on ground, GPU, deciduous forest, 29 Aug., leg. $M W \& T L$, det. $M W \& A K$; on ground, GPU, thickets, 30 Aug., leg. \& det. MW.

Lactarius pubescens (Schrad.) Fr. on ground, GPU, thickets, 30 Aug., leg. \& det. MW, $G D \& A K$; on ground, GPU, mixed forest, 30 Aug., leg. \& det. MR, TL, MP \& LK (molecular data).

Lactarius quietus (Fr.) Fr. on ground, KPU, deciduous forest, 28 Aug., leg. \& det. MR, $T L, M P \& L K$ (molecular data); on ground, GPU, thickets, 30 Aug., leg. \& det. $M W$.

Lactarius tabidus Fr. [ = L. thejogalus (Bull.) Gray] on ground, KPU, deciduous forest, 28 Aug., leg. \& det. $K K, G D, A K, M R, T L, M P \& L K$ (molecular data).

Lactarius torminosus (Schaeff.) Pers. on ground, GPU, deciduous forest, 29 Aug., leg. $\&$ det. $A K$; on ground, GPU, thickets, 30 Aug., leg. \& det. $M W$. 
Lactarius trivialis (Fr.) Fr. on ground, GPU, mixed forest, 29 Aug., leg. \& det. MR, $T L, M P \& L K$ (molecular data); $\mathbf{R}$.

Lactarius vietus (Fr.) Fr. on ground, GPU, thickets, 30 Aug., leg. GD, det. MW.

Laetiporus sulphureus (Bull.) Murrill on Carpinus betulus L., KPU, T-C, 28 Aug., vid. Cezary Werpachowski \&. $M W$.

Leccinum holopus (Rostk.) Watling on ground, KPU, deciduous forest, 28 Aug., leg. $\&$ det. $M R, T L, M P \& L K$ (molecular data); on ground, BPU, near Grobla Honczarowska, 01 Sept., $V-P$, leg. \& det. GD; V.

Leccinum scabrum (Bull.) Gray on ground, WPU, Barwik, coniferous forest 31 Aug., leg. \& det. $M W$.

Lentaria byssiseda (Pers.) Corner on log of Quercus robur, GPU, deciduous forest, 29 Aug., leg. Magdalena Maliszewska, det. BG; I.

Note. Species recorded in Poland in two localities (Wojewoda 2003; Halama, Romański 2010).

Lentinellus cochleatus (Pers.) P. Karst. var. inolens (Konrad \& Maubl.) M. Holden at the base of Corylus avellana L., GPU, deciduous forest, 29 Aug., leg. MW, det. MW $\& A K$.

Notes. Variety is often not distinguished from typical variety. Distribution in Poland is unknown.

Lentinus torulosus (Pers.) Lloyd on stump of Betula pendula Roth, KPU, T-C, 28 Aug., leg. KK, det. AK; R.

Lepiota aspera (Pers.) Quél. on ground, GPU, deciduous forest, 29 Aug., leg. \& det. $A K$; on ground, GPU, $T-C, 30$ Aug., leg. \& det. GD.

Lepiota clypeolaria (Bull.) P. Kumm. on ground, KPU, T-C, 28 Aug., leg. AK, det. $B G$; on ground, GPU, deciduous forest, 29 Aug., leg. $M \longleftarrow \& A K$, det. $B G$; on ground, GPU, roadside, 30 Aug., leg. GD, det. BG; on ground, WPU, Barwik, mixed forest, 31 Aug., leg. $A K$, det. $B G$.

Lepiota cristata (Bolton) P. Kumm. on ground, KPU, T-C, 28 Aug., leg. AK, det. BG; on ground, GPU, deciduous forest, 29 Aug., leg. $A K$, det. $B G$; on ground, GPU, Czerwone Bagno, T-C, 29 Aug., leg. GD, det. BG; on ground, GPU, roadside, 29 Aug., leg. $G D$, det. $B G$; on ground, Osowiec-Twierdza, area of the fortress, 31 Aug., leg. $G D$, det. $B G$.

Lepiota echinella Quel. \& G.E. Bernard [= L. setulosa Quél. \& G.E. Bernard] on ground, KPU, T-C, 28 Aug., leg. $A K$, det. $B G$; on ground, WPU, Barwik, thickets, 31 Aug., leg. $G D$, det. $B G ; \mathbf{E}$.

NoтE. Species recorded in Poland in several localities (Gierczyk et al. 2011c).

Lepiota echinella var. rhodorhiza (P.D. Orton) Legon \& A. Henrici, on ground KPU, T-C, 28 Aug., leg. $A K$, det. BG.

Notes. Variety is often not distinguished from typical variety. Distribution in Poland is unknown.

*Lepiota lepida Guinb. \& M. Bodin on ground, GPU, deciduous forest, 29 Aug., leg. $A K$, det. $B G$.

Notes. Species hitherto not reported in Polish literature; new for Polish mycobiota. 
Lepiota oreadiformis Velen. on ground, between the villages Kopiec and Wrotki, roadside, 28 Aug., leg. GD, det. BG; on ground, Osowiec-Twierdza, lawn, 31 Aug., leg. \& det. $A K$.

NotE. Species recorded in Poland in several localities (Gierczyk et al. 2011c).

Lepiota ventriosospora D.A. Reid on ground KPU, T-C, 28 Aug., leg. \& det. AK; on ground, GPU, deciduous forest, 29 Aug., leg. $A K \& M M$, det. $B G$; on ground, GPU, $T-C, 29$ Aug., leg. GD, det. BG.

Lepista gilva (Pers.) Pat. on ground, GPU, mixed forest, 29 Aug., leg. KK, det. AK; on ground, WPU, Barwik, coniferous forest, 31 Aug., leg. KK, det. $A K$.

Lepista irina (Fr.) H.E. Bigelow on ground, KPU, birch forest, 28 Aug., leg. \& det. $M W$.

Leucoagaricus cretaceus (Bull.) M.M. Moser on ground, Osowiec-Twierdza, lawn, 31 Aug., leg. \& det. $A K$.

Leucocortinarius bulbiger (Alb. \& Schwein.) Singer on ground, KPU, T-C, 28 Aug., leg. $A K, M W, M P \&$ det. $A K ; \mathbf{R}$.

Lycoperdon nigrescens (Pers.) Pers. on ground, KPU, T-C, 28 Aug., leg. \& det. IK.

Lycoperdon perlatum Pers. on ground, GPU, deciduous forest, 30 Aug., leg. JS, det. $M W$; on ground, WPU, Barwik, coniferous forest, 31 Aug., leg. \& det. $M W$.

Macrocystydia cucumis (Pers.) Joss. on ground, GPU, hazel thickets, 29 Aug., leg. \& det. $A K$.

Macrolepiota mastoidea (Fr.) Singer on ground, GPU, coniferous forest, 29 Aug., leg. \& det. $M W$.

Macrolepiota olivieri (Barla) Wasser on ground, KPU, T-C, 28 Aug., leg. $M W \& K K$, det. $A K$; on ground, GPU, deciduous forest, 29 Aug., leg. \& det. $A K$; on ground, GPU, $T-C, 30$ Aug., leg. $G D$, det. $B G$.

Notes. Species known in Poland from one locality (Ślusarczyk 2012). Probably not differentiated from Macrolepiota rhacodes.

Macrolepiota procera (Scop.) Singer on ground, KPU, T-C, 28 Aug., leg. \& det. MW; on ground, GPU, thickets, 30 Aug., leg. \& det. KK.

Marasmius oreades (Bolton) Fr. on ground, KPU, T-C, 28 Aug., leg. MD, det. AK; on ground, GPU, thickets, 30 Aug., leg. \& det. $M W \& A K$.

Marasmius rotula (Scop.) Fr. on fallen twigs of deciduous trees, KPU, T-C, 28 Aug., leg. Magdalena Maliszewska, $G D \& A K$, det. $A K$; on fallen twigs of deciduous trees, GPU, thickets, 30 Aug., leg. \& det. $M W$.

Megacollybia platyphylla (Pers.) Kotl. \& Pouzar on stumps of deciduous tree, KPU, T-C, 28 Aug., leg. $A K, T L \& M E$, det. $A K$; on wood of deciduous trees, GPU, deciduous forest, 29 Aug., leg. JS, det. $A K$.

Melanophyllum haematospermum (Bull.) Kreisel on ground, GPU, deciduous forest, 29 Aug., leg. \& det. AK; R.

Mycena acicula (Schaeff.) P. Kumm. on fallen twigs of deciduous trees, KPU, T-C, 28 Aug., leg. $A K \& C T$, det. $A K$. 
Mycena galericulata (Scop.) Gray on stump and log of deciduous tree, KPU, T-C, 28 Aug., leg. \& det. AK; on wood of deciduous trees, GPU, deciduous forest, 29 Aug., leg. \& det. AK.

Mycena galopus (Pers.) P. Kumm. on ground, GPU, mixed forest, 29 Aug., leg. \& det. $I K$.

Mycena haematopus (Pers.) P. Kumm. var. marginata J.E. Lange on log of deciduous tree, KPU, $T-C, 28$ Aug., leg. $A K \& G D$, det. $A K$; on wood of deciduous trees, GPU, deciduous forest, 29 Aug., leg. \& det. $A K$.

Note. Variety recorded in Poland in few localities.

Mycena megaspora Kauffman on ground among Sphagnum spp., GPU, V-P, 29 Aug., leg. $G D \& K K$, det. $A K ; \mathbf{V}$.

Mycena pelianthina (Fr.) Quél. on ground, KPU, T-C, 28 Aug., leg. MW, det. AK; on ground, GPU, deciduous forest, 29 Aug., leg. \& det. $A K ; \mathbf{I}$.

Mycena polygramma (Bull.) Gray on stump of deciduous tree, KPU, T-C, 28 Aug., leg. $A K \& M W$, det. $A K \& M W$.

Mycena pura (Pers.) P. Kumm. on ground, KPU, T-C, 28 Aug., leg. \& det. AK; ground, GPU, deciduous forest, 29 Aug., leg. \& det. $A K$; on ground, GPU, thickets, 30 Aug., leg. \& det. MW; on ground, WPU, Barwik, coniferous forest, 31 Aug., leg. \& det. AK.

Mycena rosea (Bull.) Gramberg on ground, KPU, T-C, 28 Aug., leg. AK\& GD, det. $A K$; on ground, GPU, deciduous forest, 29 Aug., leg. \& det. $A K$; on ground, WPU, Barwik, coniferous forest, 31 Aug., leg. GD, det. AK.

Mycena spirea (Fr.) Gillet on fallen twigs of deciduous tree, KPU, T-C, 28 Aug., leg. $\&$ det. $A K$.

Mycena zephirus (Fr.) P. Kumm. on litter, WPU, Barwik, coniferous forest, 31 Aug., leg. $A K \& K K$, det. $A K$.

Oligoporus ptychogaster (F. Ludw.) Falk \& O. Falck on log of Picea abies, H.Karst., GPU, Czerwone Bagno, mixed forest, 29 Aug., leg. \& det. GD; R.

Omphalina umbellifera (L.) Quél. on wood of deciduous trees among mosses, GPU, deciduous forest, 29 Aug., leg. Magdalena Maliszewska, det. AK; R.

Panaeolus alcidis M.M. Moser on deer (?) excrement, GPU, mixed forest, 29 Aug., leg. \& det. $A K$; on elk (?) excrement, GPU, thickets, 30 Aug., leg. \& det. AK.

Notes. According to literature data (Wojewoda 2003), species known from a single locality. However, it has been found in 2010 in two new localities, in BbNP and Kampinos National Park (Szczepkowski, personal comm.).

Panellus stipticus (Bull.) P. Karst. on wood of Quercus robur L., GPU, thickets, 30 Aug., leg. \& det. $M W$.

Paxillus atrotomentosus (Batsch) Fr. on wood, WPU, Barwik, coniferous forest, 31 Aug., leg. \& det. MW.

Paxillus involutus (Batsch) Fr. s.l. on ground, KPU, T-C, 28 Aug., leg. MW, IK, GD, $K K, A K \&$ det. $A K$. 
Paxillus rubicundulus P.D. Orton on ground, KPU, educational path "Brzeziny Kapickie”, birch forest, 29 Aug., leg. Magdalena Maliszewska, det. AK; R.

Note. Species recorded in Poland in several tens localities.

Phallus duplicatus Bosc on ground, GPU, T-C, 29 Aug., vid. GD; E. (Fig. 5).

Note. Species recorded in Poland in three localities (Friedrich 2011).

Phallus impudicus L. on ground, KPU, T-C, 28 Aug., leg. \& det. AK; on ground, GPU, mixed forest, 29 Aug., leg. \& det. MŁ; on ground, WPU, Barwik, coniferous forest, 31 Aug., leg. \& det. KK.

Phellinus igniarius (L.) Quél. on Salix sp., KPU, meadow, 28 Aug., vid. MW; on Salix sp., GPU, thickets, 30 Aug., leg. \& det. $M W$.

Phellinus pini (Brot.) A. Ames on Pinus sylvestris L., GPU, V-P, 29 Aug., leg. \& det. $G D, J S \& M W ; \mathbf{R}$.

Phellinus robustus (P. Karst.) Bourdot \& Galzin on Quercus robur L., KPU, T-C, 28 Aug., vid. $M W \& J S$.

Phellodon confluens (Pers.) Pouzar on ground, BPU, Grobla Honczarowska, thickets, 01 Sept., leg. $G D \& K K$, det. $B G$; Ex. (Fig. 6)

Note. Species recorded in Poland in few new localities (Kujawa 2012).

Phlebia tremellosa (Schrad.) Nakasone \& Burds. on stump and log of deciduous tree, KPU, T-C, 28 Aug., leg. \& det. AK; on wood of Corylus avellana L., GPU, mixed forest, 29 Aug., leg. \& det. MŁ.

Phleogena faginea (Fr.) Link on Populus tremula L, GPU, deciduous forest, 29 Aug., leg. TL \& Magdalena Maliszewska, det. AK; E.

Note. Species recorded in Poland in several tens localities (Szczepkowski, Chachuła 2010).

Pholiota albocrenulata (Peck) Sacc. at the base of Betula pendula Roth, KPU, T-C, 28 Aug., leg. \& det. AK; at the base of Populus sp., GPU, T-C, 29 Aug., leg. \& det. GD; E. (Fig. 7)

Pholiota cerifera (P. Karst.) P. Karst. on Salix sp., GPU, educational path Wydmy, dune, 30 Aug., leg. GD, det. BG.

Pholiota flammans (Batsch) P. Kumm. on wood of Pinus sylvestris L., GPU, deciduous forest, 29 Aug., leg. TL, det. AK; on wood, WPU, Barwik, coniferous forest, 31 Aug., leg. $K K$, det. $A K$.

Pholiota limonella (Peck) Sacc. on Alnus glutinosa (L.) Gaertn., GPU, near Góra Solistowska, mixed forest, 29 Aug., leg. GD, det. BG.

Note. Species recorded in Poland in few localities (Gierczyk et al. 2011a).

Pholiota (Kuehneromyces) mutabilis (Scop.) P. Kumm. on stumps of deciduous trees, KPU, T-C, 28 Aug., leg. \& det. AK; on wood of deciduous trees, GPU, mixed forest, 29 Aug., leg. \& det. KK\& JS.

Pholiota squarrosa (Weigel) P. Kumm. at the base of Populus sp., GPU, T-C, 29 Aug., leg. \& det. GD. 
Pholiota squarrosoides (Peck) Sacc. on log of Betula pendula Roth, GPU, near Góra Solistowska, mixed forest, 29 Aug., leg. GD, det. BG; on wood, GPU, T-C, 30 Aug., leg. \& det. GD; E. (Fig. 8)

Phyllotopsis nidulans (Pers.) Singer on log of Populus sp., GPU, T-C, 30 Aug., leg. \& det. GD; V.

Physisporinus vitreus (Pers.) P. Karst. at the base of Picea abies (L.) H. Karst., GPU, coniferous forest 29 Aug., leg. \& det. GD; R.

Piptoporus betulinus (Bull.) P. Karst. on Betula pendula Roth, KPU, T-C, 28 Aug., leg. \& det. AK; on wood of Betula pendula Roth, WPU, Barwik, coniferous forest, 31 Aug., leg. \& det. $M W$.

Pleurocybella porrigens (Pers.) Singer on ground, GPU, mixed forest, 30 Aug., leg. \& det. GD; V.

Pleurotus pulmonarius (Fr.) Quél. on wood of deciduous trees, GPU, deciduous forest, 29 Aug., leg. \& det. $K K, A K$; V.

Pluteus atricapillus (Batsch) Fayod on stump of deciduous tree, KPU, T-C, 28 Aug., leg. \& det. AK; on wood of deciduous trees, GPU, mixed forest, 29 Aug., leg. KK, det. $A K$; on wood, GPU, thickets, 30 Aug., leg. \& det. KK; on wood, WPU, Barwik, mixed forest, 31 Aug., leg. GD, det. AK.

Pluteus leoninus (Schaeff.) P. Kumm. on $\log$ of deciduous tree, KPU, T-C, 28 Aug., leg. \& det. AK; on wood of deciduous trees, GPU, mixed forest, 29 Aug., leg. \& det. $K K$; on log of Betula pendula Roth, GPU, Czerwone Bagno, coniferous forest, 29 Aug., leg. \& det. GD.

Pluteus nanus (Pers.) P. Kumm. on wood debris, KPU, T-C, 28 Aug., leg. \& det. AK; on wood, GPU, birch forest, 30 Aug., leg. GD, det. $A K$.

Pluteus plautus (Weinm.) Gillet on stump of deciduous tree, KPU, T-C, 28 Aug., leg. $M W$, det. $A K$; $\mathbf{I}$.

Note. Species recorded in Poland in several tens localities (Wojewoda 2003; Kujawa 2013).

Pluteus podospileus Sacc. \& Cub. on wood of deciduous tree, KPU, T-C, 28 Aug., leg. $M \longleftarrow$, det. $A K$.

Pluteus pouzarianus Singer var. pouzarianus on log of Picea abies (L.) H. Karst., GPU, Ur. Dęby, T-C, 29 Aug., leg. \& det. GD.

Pluteus romellii (Britzelm.) Sacc. on fallen twigs of deciduous tree, KPU, T-C, 28 Aug., leg. \& det. AK; on fallen twigs of deciduous tree, GPU, Czerwone Bagno, T-C, 29 Aug., leg. \& det. GD.

Pluteus salicinus (Pers.) P. Kumm. on wood of deciduous tree, KPU, T-C, 28 Aug., leg. MU, det. AK; on wood, WPU, Barwik, mixed forest, 31 Aug., leg. GD, det. AK.

Polyporus badius (Pers.) Schwein. on log of Betula pendula Roth, GPU, Uroczysko Dęby, T-C, 29 Aug., vid. GD.

Polyporus ciliatus Fr. on wood of deciduous tree, KPU, T-C, 28 Aug., leg. MW, det. $M W \& A K$; on wood of deciduous trees, GPU, mixed forest, 29 Aug., leg. $M W$, det. $M W \& A K$. 

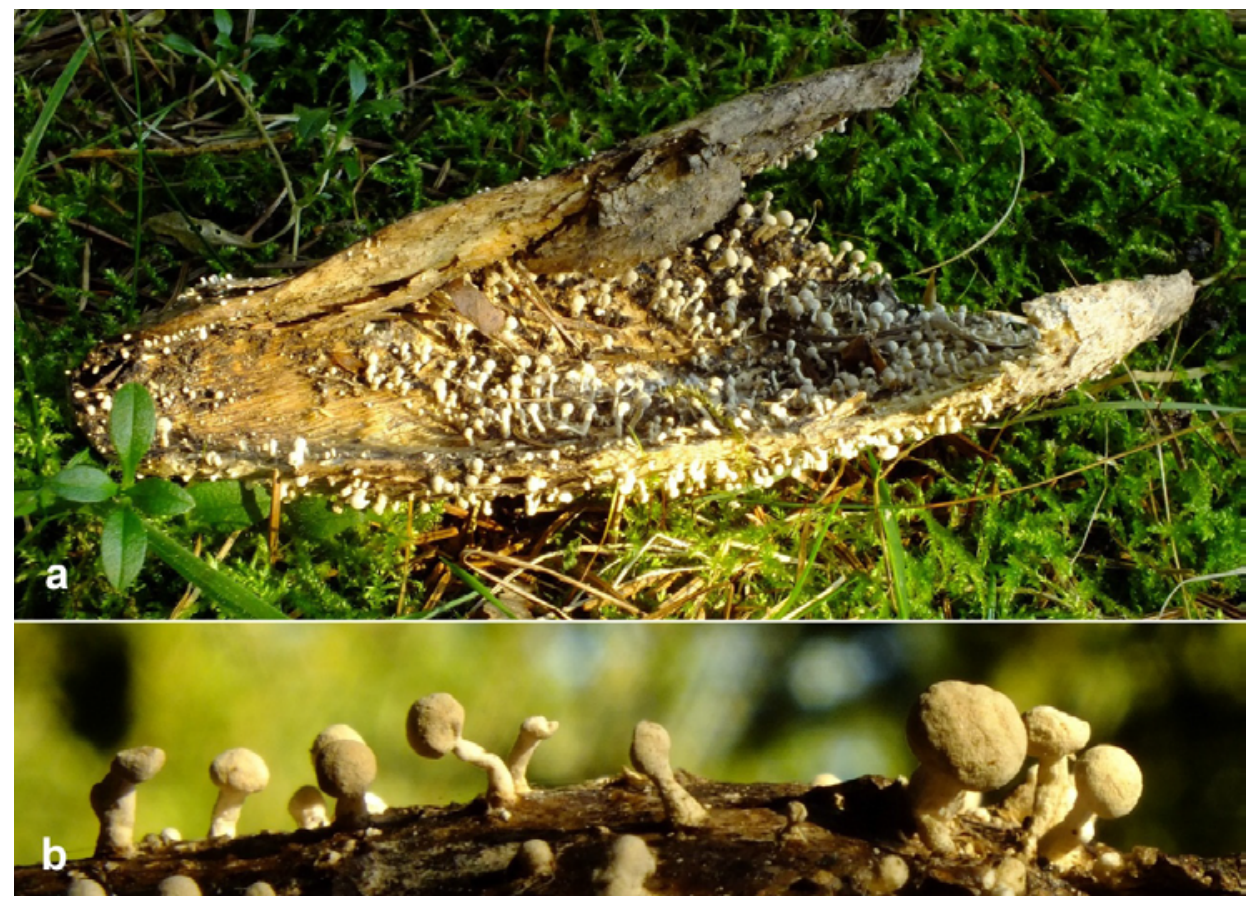

Fig. 2. Onygena equina, keratinophilic species decomposing animal remnants: a - general view of substrate, presumably hoof of elk, a - fruitbodies, magnified. Species threatened due to its rarity ( $\mathrm{R}$ category).

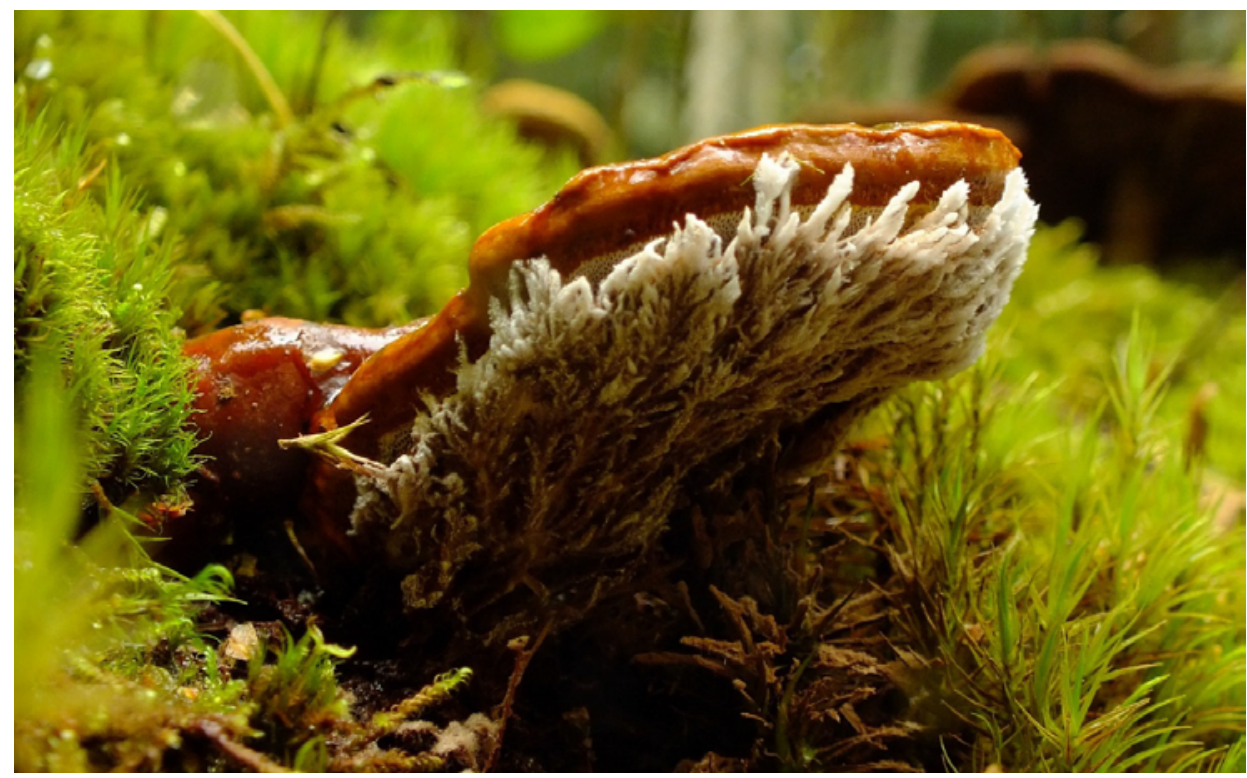

Fig. 3. Ganoderma lucidum, fruitbody with anamorphic state visible on its lower surface; threatened (R category) and protected in Poland. 


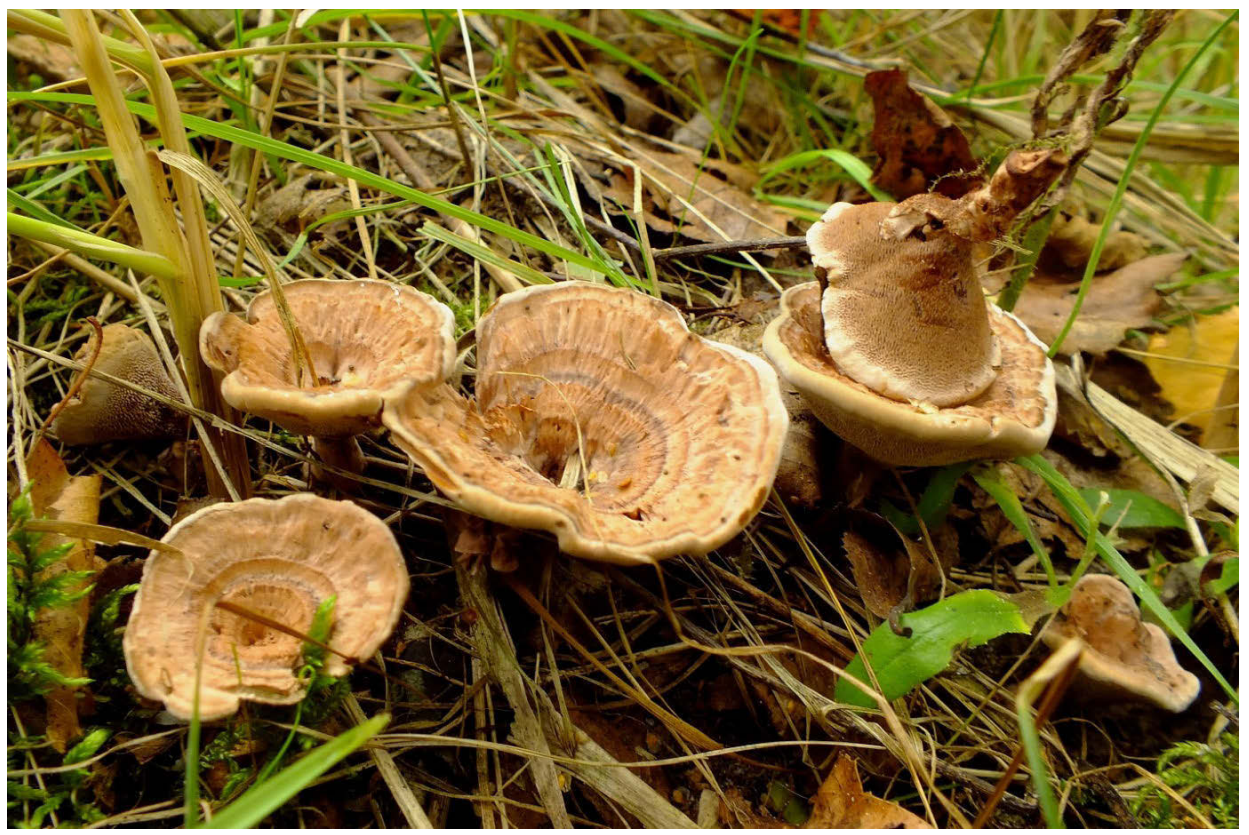

Fig. 4. Hydnellum concrescens, mycorrhizal species, threatened (E category) and protected in Poland.

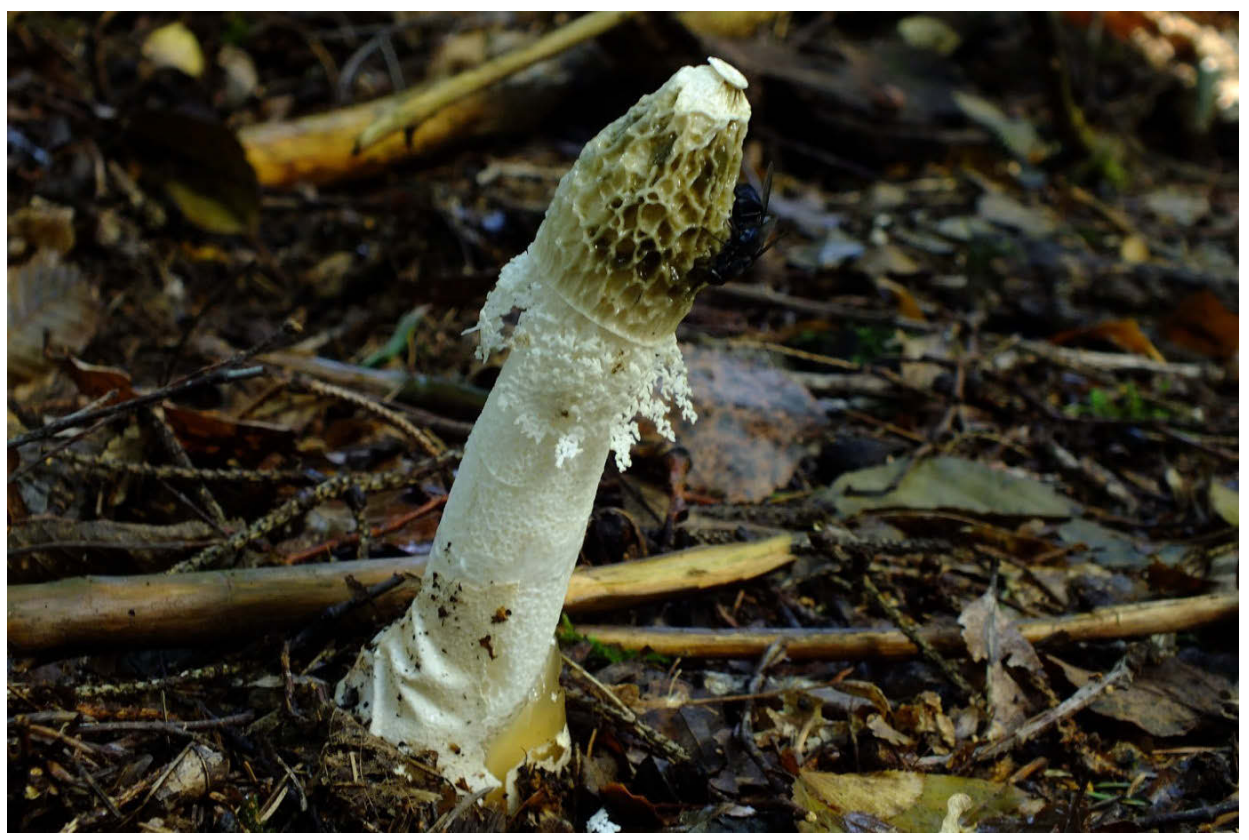

Fig. 5. Phallus duplicatus, fruitbody with characteristic short, net-like indusium hanging from the margin of cap-like part of the receptacle; species very rare in Poland and threatened (E category). 


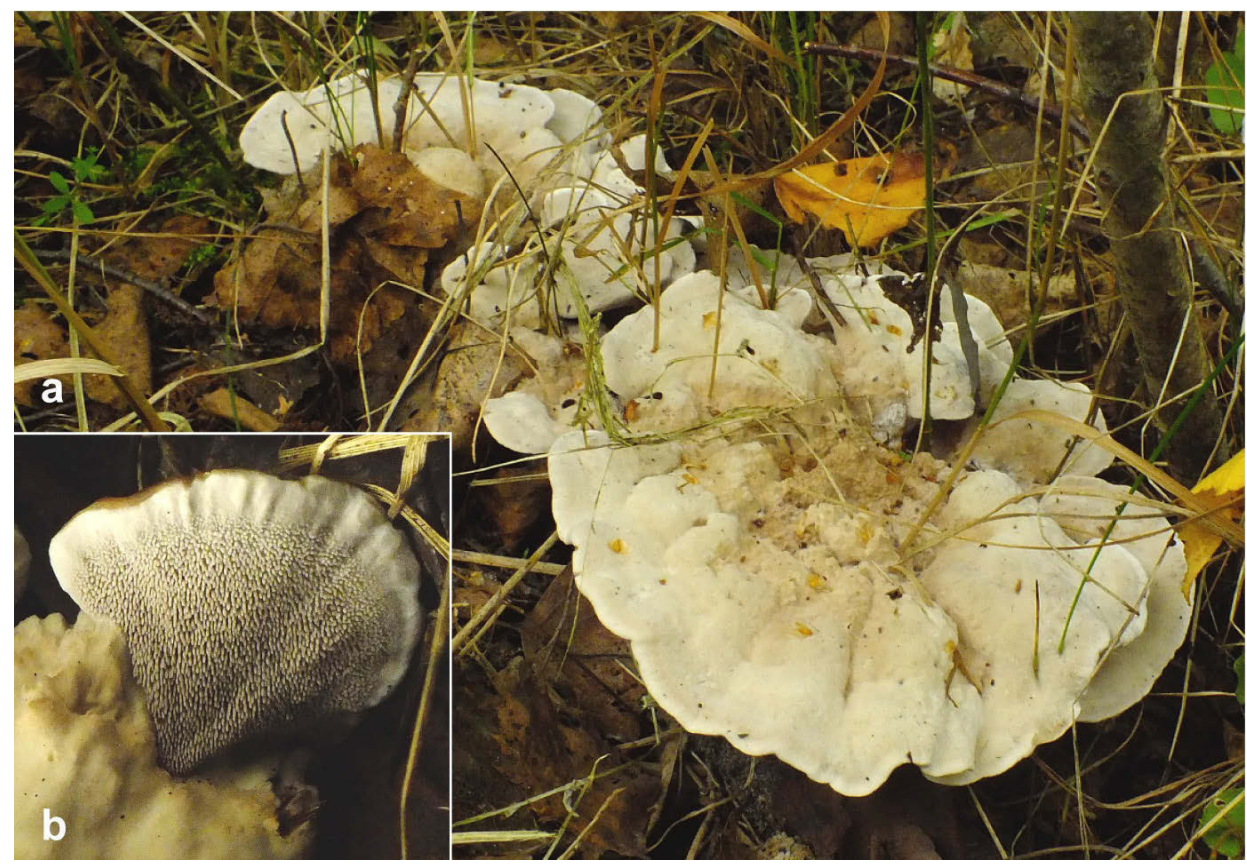

Fig. 6. Phellodon confluens: a - general view of fruitbodies in natural habitat, $\mathrm{b}$ - spines with hymenial layer at the fruitbody lower surface. Species regarded as extinct in Poland (Ex category).

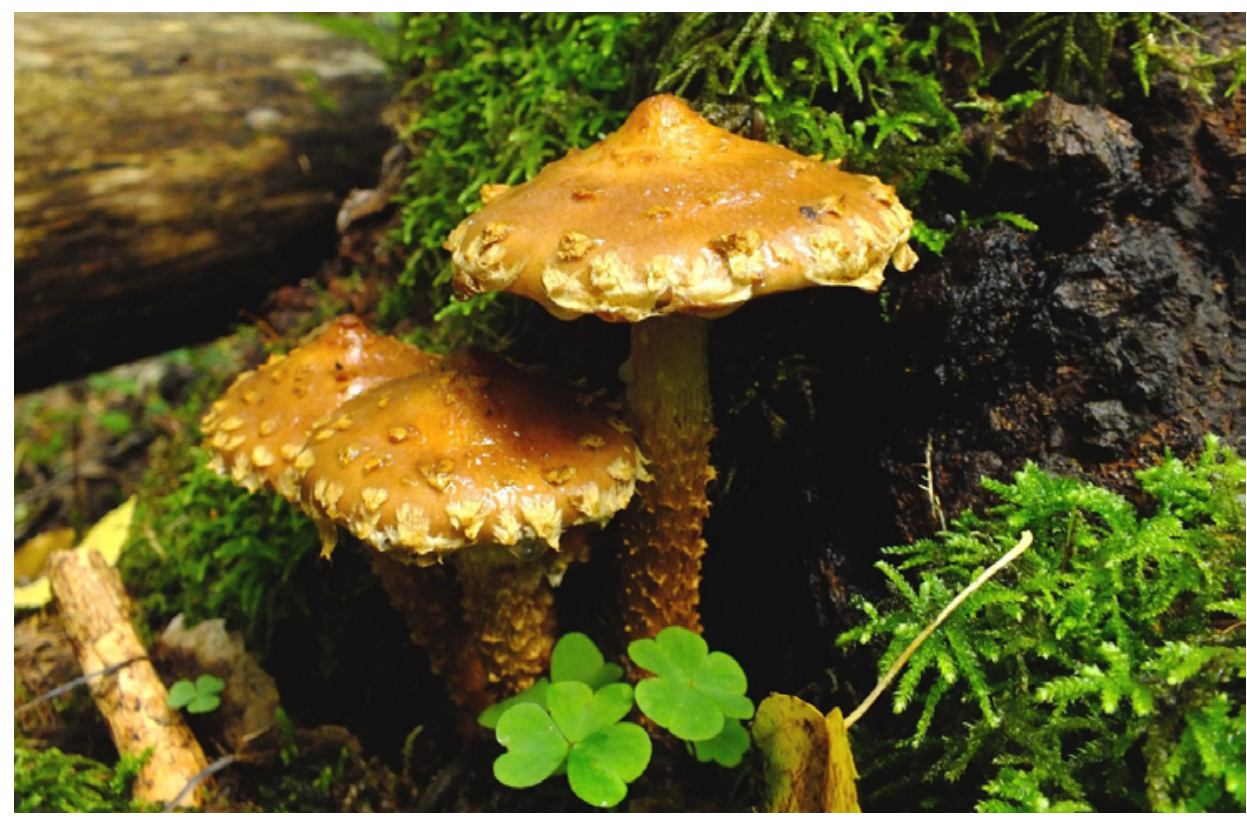

Fig. 7. Pholiota albocrenulata, saprobe or parasite growing at the base of deciduous and coniferous trees; threatened (E category). 


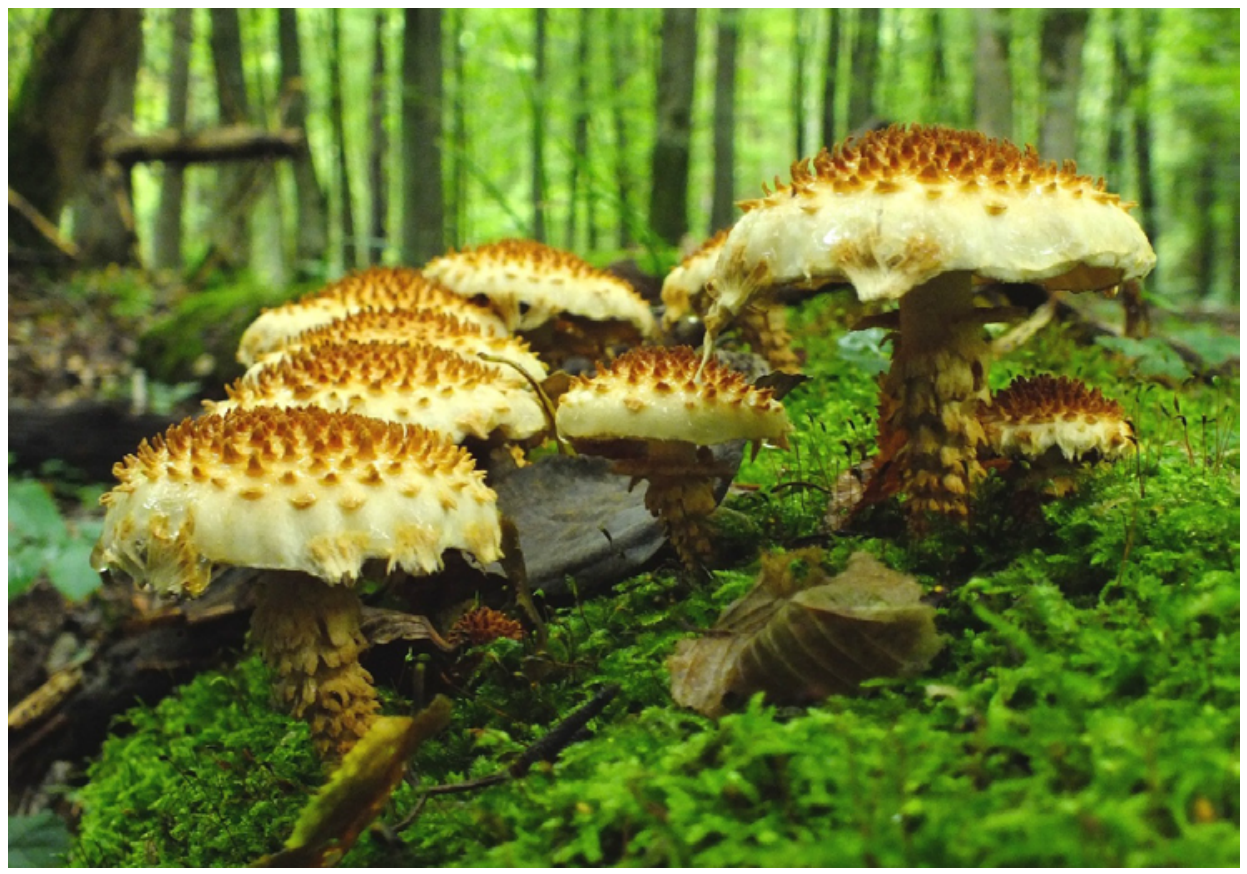

Fig. 8. Pholiota squarrosoides, saprobe decomposing wood of deciduous trees, characteristic to natural plant communities; endangered in Poland (E category).

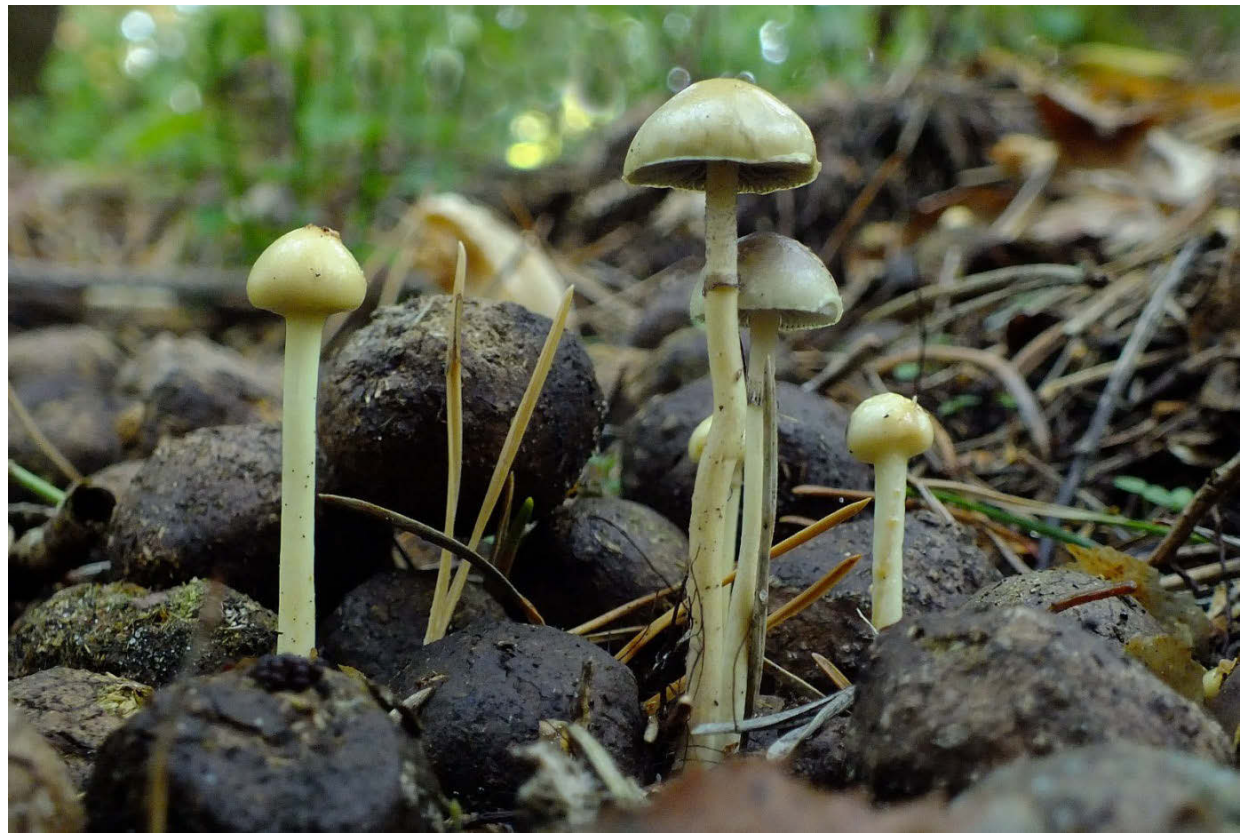

Fig. 9. Stropharia alcis, very similar to S. semiglobata, distinguishable by the shorter and narrower basidiospores; species new for Poland. 
Polyporus squamosus (Huds.) Fr. on wood of Salix sp. (?), Osowiec-Twierdza, area of the fortress, 31 Aug., leg. \& det. MW.

Polyporus tuberaster (Jacq.) Fr. on wood of Betula pendula Roth, KPU, T-C, 28 Aug., leg. $M W \& D S$, det. $M W$; on wood of deciduous trees, KPU, educational path "Brzeziny Kapickie", birch forest, 29 Aug., leg. Magdalena Maliszewska, det. AK; on wood of deciduous trees, GPU, deciduous forest, 29 Aug., leg. \& det. AK; R.

Psathyrella candolleana (Fr.) Maire on wood of deciduous trees, KPU, educational path "Brzeziny Kapickie", birch forest, 29 Aug., leg. Magdalena Maliszewska, det. AK; on ground, WPU, Barwik, deciduous forest, 31 Aug., leg. GD, det. AK.

Psathyrella gordonii (Berk. \& Broome) A. Pearson \& Dennis on ground, Goniądz, riverside, 31 Aug., leg. $M W$, det. $B G$.

Psathyrella spadicea (Schaeff.) Singer on the base of Betula pendula Roth, GPU, mixed forest, 29 Aug., leg. \& det. AK.

Pseudohydnum gelatinosum (Scop.) P. Karst. on wood of Picea abies (L.) H. Karst., GPU, T-C, 30 Aug., vid. GD.

Pseudomerulius aureus (Fr.) Jülich on wood of Pinus sylvestris L., GPU, V-P, 29 Aug., leg. \& det. GD; R.

Pycnoporellus fulgens (Fr.) Donk on Picea abies (L.) H. Karst., GPU, T-C, 29 Aug., leg. \& det. GD; V.

Pycnoporus cinnabarinus (Jacq.) P. Karst. on wood of Betula pendula Roth, GPU, thickets, 30 Aug., leg. \& det. GD; R.

Ramaria eumorpha (P. Karst.) Corner on ground, GPU, T-C, 30 Aug., leg. \& det. GD. Ramaria flaccida (Fr.) Bourdot on ground, GPU, mixed forest, 29 Aug., leg. KK, det. $A K$.

Ramaria stricta (Pers.) Quél. on ground, KPU, deciduous forest, 28 Aug., leg. \& det. $M R, T L, M P \& L K$ (molecular data); on ground, GPU, mixed forest, 30 Aug., leg. \& det. $M R, T L, M P \& L K$ (molecular data).

Rhodocollybia butyracea (Bull.) Lennox f. asema (Fr.) Antonín, Halling \& Noordel on ground, WPU, Barwik, coniferous forest, 31 Aug., leg. GD, det. AK.

Rhodocollybia filamentosa (Velen.) Antonín on ground, WPU, Barwik, coniferous forest, 31 Aug., leg. $M W$, det. AK.

Rickenella fibula (Bull.) Raithelh. on stump among mosses, KPU, T-C, 28 Aug., leg. $G D \& J S$, det. $G D$; on wood of deciduous trees among mosses, GPU, deciduous forest, 29 Aug., leg. \& det. $M W \& A K$.

Rickenella setipes (Fr.) Raithelh. on wood of deciduous trees among mosses, GPU, deciduous forest, 29 Aug., leg. Magdalena Maliszewska, det. AK.

Rozites caperata (Pers.) P. Karst. on ground, GPU, deciduous forest, 29 Aug., leg. \& det. $M W$; on ground, GPU, mixed forest, 30 Aug., vid. GD.

Russula aeruginea Lindbl. on ground, KPU, deciduous forest, 28 Aug., leg. \& det. $M R, T L, M P \& L K$ (molecular data); on ground, GPU, V-P, 29 Aug., leg. \& det. $M W$; on ground, GPU, thickets, 30 Aug., leg. \& det. $M W$; on ground, GPU, mixed forest, 
30 Aug., leg. \& det. $M R, T L, M P \& L K$ (molecular data); on ground, WPU, Barwik, coniferous forest, 31 Aug., leg. \& det. $M W$.

Russula claroflava Grove on ground under Betula pendula Roth, KPU, T-C, 28 Aug., leg. \& det. $A K$; on ground, GPU, deciduous forest, 29 Aug., leg. \& det. $A K$; on ground, GPU, thickets, 30 Aug., leg. \& det. $A K$.

Russula clavipes Velen. [ $=R$. elaeodes (Bres.) Bon] on ground, GPU, mixed forest, 29 Aug., leg. \& det. $M R, T L, M P \& L K$ (molecular data).

Notes. Species recorded in Poland in two localities.

Russula graveolens Romell on ground, KPU, deciduous forest, 28 Aug., leg. \& det. $M R, T L, M P \& L K$ (molecular data).

Note. Species recorded in Poland in three localities (Wojewoda 2003; Karasiński 2009; Kujawa, Gierczyk 2011).

Russula ochroleuca (Pers.) Fr. on ground, GPU, deciduous forest, 29 Aug., leg. \& det. $I K$.

Russula pectinatoides Peck on ground, KPU, T-C, 28 Aug., leg. MW, det. MŁ; on ground, KPU, deciduous forest, 28 Aug., leg. \& det. $M R, T L, M P \& L K$ (molecular data); on ground, GPU, mixed forest, 30 Aug., leg. \& det. MR, TL, MP \& LK (molecular data).

Russula sanguinea (Bull.) Fr. on ground, GPU, mixed forest, 30 Aug., leg. \& det. MR, $T L, M P \& L K$ (molecular data).

Russula velenovskyi Melzer \& Zvara on ground, KPU, deciduous forest, 28 Aug., leg. $\&$ det. $M R, T L, M P \& L K$ (molecular data); on ground, GPU, mixed forest, 30 Aug., leg. \& det. $M R, T L, M P \& L K$ (molecular data).

Russula vesca Fr. on ground, GPU, deciduous forest, 29 Aug., leg. \& det. AK; on ground, KPU, deciduous forest, 28 Aug., leg. \& det. $M R, T L, M P \& L K$ (molecular data); on ground, GPU, mixed forest, 30 Aug., leg. \& det. MR, TL, MP \& LK (molecular data).

Russula vinosa Lindblad on ground, GPU, mixed forest, 29 Aug., leg. \& det. MR, $T L$, $M P \& L K$ (molecular data).

Schizophyllum commune Fr. on log of deciduous tree, KPU, T-C, 28 Aug., leg. \& det. $M W$.

Scleroderma areolatum Ehrenb. on ground, KPU, T-C, 28 Aug., leg. \& det. MW; on ground, GPU, deciduous forest, 29 Aug., leg. \& det. $M W$; on ground, GPU, thickets, 30 Aug., leg. GD, det. $A K$.

Scleroderma bovista Fr. on ground, KPU, T-C, 28 Aug., leg. \& det. AK.

Scleroderma citrinum Pers. on ground, GPU, T-C, 28 Aug., leg. \& det. GD.

Scleroderma verrucosum (Bull.) Pers. on ground, KPU, deciduous forest, 28 Aug., leg. $\&$ det. $M R, T L, M P \& L K$ (molecular data).

Stereum hirsutum (Willd.) Gray on wood of deciduous tree, KPU, T-C, 28 Aug., leg. $A K \& J S$, det. $A K$.

Stereum sanguinolentum (Alb. \& Schwein.) Fr. on wood of Pinus sylvestris L., GPU, deciduous forest, 29 Aug., leg. \& det. $J S \& M W$. 
Stereum subtomentosum Pouzar on fallen twigs and log of deciduous tree, KPU, T-C, 28 Aug., leg. \& det. GD; on wood of deciduous trees, GPU, deciduous forest, 29 Aug., leg. \& det. $M \longleftarrow, J S \& A K$.

Stropharia aeruginosa (Curtis) Quél. on wood, GPU, T-C, 30 Aug., leg. \& det. GD.

*Stropharia alcis Kytöv. on elk excrement, GPU, Czerwone Bagno, V-P, 29 Aug., leg. GD, det. BG; on elk excrement, GPU, T-C, 30 Aug., leg. GD, det. BG. (Fig. 9)

Note. Species hitherto not reported in Polish literature, known also from Kampinos National Park (Kujawa, unpubl. data).

Stropharia squamosa (Pers.) Quél. on ground, GPU, T-C, 30 Aug., leg. \& det. GD; I. Suillus bovinus (L.) Roussel, on ground, GPU, thickets, 30 Aug., leg. \& det. MR, TL, $L K \& M P$ (molecular data).

Suillus granulatus (L.) Roussel on ground, GPU, thickets, 30 Aug., leg. KK, det. AK. Thelephora caryophyllea (Schaeff.) Fr. on ground, GPU, thickets, 30 Aug., leg. \& det. $M W$; on ground, OPU, Sośnia, thickets, 30 Aug., leg. \& det. GD; V.

Thelephora terrestris Ehrh. on wood of deciduous trees, GPU, mixed forest, 29 Aug., leg. $K K$, det. $A K$.

Trametes hirsuta (Wulfen) Pilát on stump of deciduous tree, KPU, T-C, 28 Aug., leg. $\&$ det. $A K$.

Tremella foliacea Pers. on twigs of Betula pendula Roth, GPU, T-C, 30 Aug., leg. \& det. GD; I.

Tremella mesenterica Retz. on twigs of Betula pendula Roth, KPU, T-C, 28 Aug., leg. $M W \& J S$, det. $M W$.

Trichaptum abietinum (Dicks.) Ryvarden on wood of coniferous trees, GPU, deciduous forest, 29 Aug., leg. \& det. AK.

Trichaptum biforme (Fr.) Ryvarden on wood of deciduous trees, GPU, deciduous forest, 29 Aug., leg. \& det. JS \& KK; $\mathbf{R}$.

Trichaptum fuscoviolaceum (Ehrenb.) Ryvarden on wood of coniferous trees, GPU, mixed forest, 29 Aug., leg. \& det. KK; on wood of Pinus sylvestris L., GPU, thickets, 30 Aug., leg. JS, det. AK.

Tricholoma scalpturatum (Fr.) Quél. on ground, GPU, thickets, 30 Aug., leg. \& det. AK. Tricholoma sulphureum (Bull.) P. Kumm. on ground, GPU, deciduous forest, 29 Aug., leg. \& det. JS.

Tricholomopsis rutilans (Schaeff.) Singer on ground, WPU, Barwik, coniferous forest 31 Aug., leg. KK, det. AK.

Tulostoma kotlabae Pouzar on ground, GPU, educational path Wydmy, dune, 30 Aug., leg. \& det. GD; P, E.

Note. Protected species recorded in Poland in two localities (Wojewoda 2003; Tomaszewska et al. 2011).

Tylopilus felleus (Bull.) P. Karst. on ground, GPU, mixed forest, 29 Aug., leg. \& det. GD.

Typhula erythropus (Pers.) Fr. on litter, GPU, deciduous forest, 29 Aug., leg. \& det. $G D$. 
Xerocomus badius (Fr.) Kühner ex E.-J. Gilbert on ground, GPU, mixed forest, 29 Aug., leg. \& det. MW; on ground, GPU, thickets, 30 Aug., leg. \& det. KK.

*Xerocomus cisalpinus Simonini, H. Ladurner \& Peintner, on ground, GPU,T-C, 28 Aug., leg. \& det. $M R, T L, L K \& M P$.

Note. Species hitherto not reported in Polish literature, known from Kampinos National Park (Gierczyk, personal comm.).

*Xerocomus fennicus (Harmaja) H. Ladurner \& Simonini on ground, GPU, mixed forest, 30 Aug., leg. \& det. MR, TL, MP \& LK (molecular data).

NoтE. Species hitherto not reported in Polish literature, known from Kampinos National Park (Gierczyk, personal comm.).

Xerocomus parasiticus (Bull.) Quél. on Scleroderma citrinum Pers., GPU, T-C, 29 Aug., leg. \& det. GD; $\mathbf{P}, \mathbf{R}$.

Xerocomus pascuus (Pers.) Krombh. s.l. on ground, KPU, T-C, 28 Aug., leg. DŚ \& JS, det. IK; on ground, GPU, deciduous forest, 29 Aug., leg. \& det. $M D \& A K$; on ground, WPU, Barwik, coniferous forest, 31 Aug., leg. \& det. MW.

Xerocomus subtomentosus (L.) Quél. on ground, GPU, mixed forest, 29 Aug., leg. \& det. $A K$; on ground, WPU, Barwik, coniferous forest 31 Aug., leg. \& det. KK.

Xeromphalina campanella (Batsch) Kühner \& Maire on wood of coniferous trees, GPU, deciduous forest, 29 Aug., leg. \& det. $M W, M \longleftarrow, A K, J S$.

Xylobolus frustulatus (Pers.) P. Karst. on wood of Quercus robur L., GPU, T-C, 29 Aug., leg. \& det. GD; on wood of Quercus robur, GPU, T-C, 30 Aug., vid. GD; V. Note. Species recorded in Poland in several localities (Stasínska 2008; Kujawa 2012).

\section{FINAL REMARKS}

This short-term survey represents the first major investigation of the macromycetes from the BbNP and has revealed 289 taxa, with $78 \%$ of the species being newly reported and thus significantly extending the list of species known to be from this area. We are aware that a four-day survey, covering only selected habitats and localities, does not reflect the real species richness and taxonomic diversity of macromycetes in the BbNP. Even based on such preliminary data we can suppose that the number of fungi occurring in the BbNP area is several times greater than currently estimated. Therefore, a long-term inventory is needed in both the entire park area and in selected, interesting and rarely studied habitats.

Key outcomes:

- Finding of five species new in Polish mycobiota (i.e. Amanita olivaceogrisea, Lepiota lepidea, Stropharia alcis, Xerocomus cisalpinus, X. fennicus) and 16 taxa very rarely recorded in Poland (i.e. Amanita argentea, Conocybe microspora, Coprotus luteus, Coprinus ellisii, C. romagnesianus, Cortinarius illibatus, Crepidotus croccophyllus, Inocybe appendiculata, Lentaria byssiseda, Panaeolus alcidis, Phallus duplicatus, Phellodon confluens, Russula clavipes, R. graveolens, Stropharia alcis and Tulostoma kotlabae). 
- Extending the knowledge of the biogeography of 59 fungi species currently protected by law (P) and referred to as threatened in Poland (Ex, E, V, R, I - category): Antrodiella serpula (R), Asterophora lycoperdoides (R), Calocera furcata (R), Clavicorona pyxidata (V), Climacocystis borealis (R), Clitocybe hydrogramma (R), Coprinus niveus (R), Cortinarius violaceus (V), Cystodermella granulosum (I), Disciseda bovista (E), Exidia glandulosa (R), Fistulina hepatica (P; R), Ganoderma lucidum (P; R), Geastrum fimbriatum (P; R), G. minimum (P; E), G. rufescens (P; E), Gloeoporus dichrous (E), Gomphidius glutinosus (R), Gyroporus castaneus (R), G. cyanescens (R), Helvella lacunosa (R), Hydnellum concrescens $(\mathrm{P} ; \mathrm{E})$, Inonotus obliquus (P; R), Ischnoderma benzoinum (V), Lactarius trivialis (R), Leccinum holopus (V), Lentaria byssiseda (I), Lentinus torulosus (R), Lepiota echinella (E), Leucocortinarius bulbiger (R), Melanophyllum haematospermum (R), Mycena megaspora (V), M. pelianthina (I), Oligoporus ptychogaster (R), Omphalina umbelifera (R), Onygena equuina (R), Paxillus rubicundulus (R), Phallus duplicatus (E), Phellinus pini (R), Phellodon confluens (Ex), Phleogena faginea (E), Pholiota albocrenulata (E), Ph. squarrosoides (E), Phyllotopsis nidulans (V), Physisporinus vitreus (R), Pleurocybella porrigens (V), Pleurotus pulmonarius (R), Pluteus plautus (I), Polyporus tuberaster (R), Pseudomerulis aureus (R), Pycnoporellus fulgens (V), Pycnoporus cinnabarinus (R), Stropharia squamosa (I), Thelephora caryophyllea (V), Tremella foliacea (I), Trichaptum biforme (R), Tulostoma kotlabae (P; E), Xerocomus parasiticus (P; R), Xylobolus frustulatus (V).

Collectively, both data on macro- and micromycetes (Ruszkiewicz-Michalska et al. 2012, this issue) point out the importance of the Biebrza Valley ecosystem in the biodiversity of fungi in particular and possibly in overall biodiversity and its role as an invaluable preserve for the northeast part of Poland. We hope that our research results will be useful in inclusion of fungi into the future management plans in the Biebrza National Park.

Acknowledgments. We are deeply grateful to Mr. Andrzej Grygoruk (who at the time of our stay was the acting managing director) as well as to Ms. Magdalena Maliszewska, Ms. Katarzyna Nowicka, Mr. Cezary Werpachowski and Mr. Krzysztof Frąckiel, and other employees of the Biebrza National Park for collecting fungi, offering their facilities, helping in the implementation of our research and creating the very friendly atmosphere during our visit. This study was partly supported by the Institute of Dendrology PAS in Kórnik and by Ministry of Science and Higher Education (NN 305304540). Sincere thanks are also addressed to anonymous reviewers for considerable remarks on the text.

\section{REFERENCES}

Anonymous 1968. Compte-rendu du IV-éme Congrés des Mycologues Europeéns Warszawa 1966. Acta Mycol. 4 (2): 181-198.

Bas C., Kuyper T.W., Noordeloos M.E., Vellinga E.C (eds). 1990. Flora Agaricina Neerlandica. Critical monograps on families of agarics and boleti occurring in the Netherlands. 2. Pleurotaceae, Pluteaceae, Tricholomataceae (1). A.A. Balkema, Rotterdam, $160 \mathrm{pp}$.

Bas C., Kuyper T.W., Noordeloos M.E., Vellinga E.C (eds). 1995. Flora Agaricina Neerlandica. Critical monograps on families of agarics and boleti occurring in the Netherlands. 3. Tricholomataceae (2). A.A. Balkema, Rotterdam, 183 pp. 
Bas C., Kuyper T.W., Noordeloos M.E., Vellinga E.C (eds). 1998. Flora Agaricina Neerlandica. Critical monograps on families of agarics and boleti occurring in the Netherlands. 1. Entolomataceae. A.A. Balkema, Rotterdam, 182 pp.

Bas C., Kuyper T.W., Noordeloos M.E., Vellinga E.C (eds). 1999. Flora Agaricina Neerlandica. Critical monograps on families of agarics and boleti occurring in the Netherlands. 4. Tricholomataceae. A.A. Balkema, Rotterdam, 194 pp.

Bujakiewicz A. 2011. Macrofungi in the Alnetum incanae association along Jaworzyna and Skawica river valleys Western Carpathians. Pol. Bot. J. 56 (2): 267-285.

Bujakiewicz A., Kujawa A. 2010. Macrofungi in selected reserves of Białowieża Forest. Parki nar. Rez. Przyr. 29 (1): 3-26. [in Polish with English summary].

Chmiel M.A. 2006. Checklist of Polish larger Ascomycetes. (In:) Z. Mirek (ed.). Biodiversity of Poland 8. W. Szafer Institute of Botany, Polish Academy of Sciences, Kraków, 152 pp.

Dahlberg A., Genney D.R., Heilmann-Clausen J. 2011. Developing a comprehensive strategy for fungal conservation in Europe: current status and future needs. Fungal Ecol. 3: 50-64. doi:10.1016/j. funeco.2009.10.004

Dyrcz A., Werpachowski C. (eds). 2005. Przyroda Biebrzańskiego Parku Narodowego. Monografia. Wyd. Biebrzański Park Narodowy, Osowiec-Twierdza, 440 pp.

Flisińska Z. 2004. Grzyby Lubelszczyzny. Wielkoowocnikowe podstawczaki (Basidiomycetes). 2. [Fungi in the Lublin Region. Macromycetes (Basidiomycetes) 2.]. Lubelskie Towarzystwo Naukowe, Lublin, 530 pp. [in Polish with English summary].

Friedrich S. 2011. New locations of threatened and protected Gasteromycetes s.1. in Northwestern Poland. Polish J. Environ. Stud. 20 (3): 559-564.

Gierczyk B., Halama M., Kujawa A., Zalas M. 2011a. Pholiota limonella - the overlooked species? Fragm. Flor. Geobot. Polonica 18 (1): 147-158. [in Polish with English summary].

Gierczyk B., Kujawa A., Pachlewski T., Szczepkowski A., Wójtowski M. 2011b. Rare species of the genus Coprinus Pers. s. lato. Acta Mycol. 46 (1): 27-73.

Gierczyk B., Kujawa A., Szczepkowski A., Chachuła P. 2011c. Rare species of Lepiota and related genera. Acta Mycol. 46 (2): 137-178.

Halama M. 2009. The first locality of Amanita argentea Huijsman (Agaricales, Basidiomycota) in southwestern Poland. Opole Scientific Society Nature Journal 42: 43-48.

Halama M., Romański M. 2010. Grzyby makroskopijne (macromycetes). (In:) L. Krzysztofiak (ed.). Śluzowce Myxomycetes, grzyby Fungi i mszaki Bryophyta Wigierskiego Parku Narodowego. Przyroda Wigierskiego Parku Narodowego. Seria naukowa. Stowarzyszenie "Człowiek i Przyroda", Suwałki: 87-201. [in Polish].

Index Fungorum, retrieved 10.12.2012 at www.indexfungorum.org

Karasiński D. 2009. Macrofungi of the „Ochojec” Nature Reserve. (In:) J.B. Parusel (ed.). Rezerwat przyrody „Ochojec” w Katowicach (Górny Śląsk). Monografia naukowo-dydaktyczna. Centrum Dziedzictwa Przyrody Górnego Śląska, Katowice: 86-103. [in Polish].

Karasiński D., Kujawa A., Piątek M., Ronikier A., Wołkowycki M. 2009. Contribution to biodiversity assessment of european primeval forests: new records of rare fungi in the Białowieża Forest. Pol. Bot. J. 54 (1): 55-97.

Kirk P.M., Cannon P. F., Minter D. W., Stalpers J. A. (eds). 2008. Ainsworth \& Bisby's Dictionary of the Fungi, $10^{\text {th }}$ ed. CABI, Wallingford, $759 \mathrm{pp}$.

Knudsen H., Vesterholt J. (eds.) 2008. Funga Nordica. Agaricoid, boletoid and cyphelloid genera. Nordsvamp, Copenhagen, $557 \mathrm{pp}$.

Kolanko K. 2005. Lichens of Biebrza National Park and its environs. (In:) A. Dyrcz, C. Werpachowski (eds). Przyroda Biebrzańskiego Parku Narodowego. Monografia. Wyd. Biebrzański Park Narodowy, Osowiec-Twierdza: 149-160 [in Polish with English summary].

Kujawa A. 2012. Grzyby makroskopijne Polski w literaturze mikologicznej. (In:) M. Snowarski Atlas grzybów Polski. Retrieved 15.12.2012, from the database at http://www.grzyby.pl/grzyby-makroskopijnePolski-w-literaturze-mikologicznej.htm [in Polish].

Kujawa A., Gierczyk B. 2011. Register of protected and endangered fungi species in Poland. Part V. A list of species recorded in 2009. Przegląd Przyrodniczy 22 (4): 16-68. [in Polish with English summary].

Leski T., Pietras M., Rudawska M. 2010. Ectomycorrhizal fungal communities of pedunculate and sessile oak seedlings from bare-root forest nurseries. Mycorrhiza 20: 179-190. doi:10.1007/s00572-0090278-6 
Mirek Z., Piękoś-Mirkowa H., Zając A., Zając M. (eds). 2002. Flowering plants and pteridophytes of Poland. A checklist. (In:) Z. Mirek (ed.). Biodiversity of Poland 1. W. Szafer Institute of Botany, Polish Academy of Sciences, Kraków, 442 pp.

Mleczko P. 2004. Mycorrhizal and saprobic macrofungi of two zinc wastes in southern Poland. Acta Biol. Cracov. Ser. Bot. 46: 25-38.

Mułenko W., Majewski T., Ruszkiewicz-Michalska M. (eds). 2008. A preliminary checklist of micromycetes in Poland. (In:) Z. Mirek (ed.). Biodiversity of Poland 9. W. Szafer Institute of Botany, Polish Academy of Sciences, Kraków, 752 pp.

Noordeloos M.E., Kuyper T.W., Vellinga E.C (eds). 2001. Flora Agaricina Neerlandica. Critical monograps on families of agarics and boleti occurring in the Netherlands. Vol. 5 Agaricaceae. A.A. Balkema Publishers, Rotterdam, 169 pp.

Noordeloos M.E., Kuyper T.W., Vellinga E.C (eds). 2005. Flora Agaricina Neerlandica. Critical monograps on families of agarics and boleti occurring in the Netherlands. Vol. 6. Taylor \& Francis Group, 226 pp.

Ronikier A. 2012. Fungi of the Sarnia Skała massif in the Tatra Mountains (Poland). Pol. Bot. Studies 28: 1-293.

Rudawska M., Leski T., Stasińska M. 2011. Species and functional diversity of ectomycorrhizal fungal communities on Scots pine (Pinus sylvestris L.) trees on three different sites. Annals of Forest Science 68: 5-15. doi:10.1007/s13595-010-0002-x

Ruszkiewicz-Michalska M., Tkaczuk C., Dynowska M., Sucharzewska E., Szkodzik J., Wrzosek M. 2012. Preliminary studies of fungi in the Biebrza National Park (NE Poland). I. Micromycetes. Acta Mycol. 47 (2): 213-234.

Rutkowski L. 2004. Klucz do oznaczania roślin naczyniowych Polski niżowej. PWN, Warszawa, 814 pp. [in Polish].

Stasińska M. 2008. Contribution to chorology of Xylobolus frustulatus in Poland. Acta Mycol. 43 (2): 167-171.

Szczepkowski A., Chachuła P. 2010. New localities and new hosts of Phleogena faginea (Fr.) Link in Poland. Parki nar. Rez. Przyr. 29 (1): 93-98. [in Polish with English summary].

Ślusarczyk T. 2012. Robinia forests as a refuge for rare and threatened macromycetes. Przegląd Przyrodniczy 23 (2): 11-41 [in Polish with English summary].

Tomaszewska A., Łuszczyński J., Łuszczyńska B., Jaworska J. 2011. Taxonomic notes on the Polish Tulostoma species. Acta Mycol. 46 (2): 179-186.

Wojewoda W. 2003. Checklist of Polish larger Basidiomycetes. (In:) Z. Mirek (ed.). Biodiversity of Poland 7. W. Szafer Institute of Botany, Polish Academy of Sciences, Kraków, 812 pp. [in Polish with English summary].

Wojewoda W., Ławrynowicz M. 2006. Red list of the macrofungi in Poland. (In:) Z. Mirek, K. Zarzycki, W. Wojewoda, Z. Szeląg (eds). Red list of plants and fungi in Poland. W. Szafer Institute of Botany, Polish Academy of Sciences, Kraków: 53-70. 
Wstępne badania grzybów w Biebrzańskim Parku Narodowym.

II. Grzyby wielkoowocnikowe

\begin{abstract}
Streszczenie
Prezentowane dane są wynikiem badań prowadzonych w dniach od 28 sierpnia do 1 września 2012 w ramach sesji terenowej Polskiego Towarzystwa Mykologicznego. Celem była wstępna inwentaryzacja grzybów wielkoowocnikowych występujących na terenie Biebrzańskiego Parku Narodowego (BbPN), terenu niemal zupełnie nie poznanego pod względem mykobioty. W artykule zamieszczono wykaz zidentyfikowanych 289 taksonów (gatunków, odmian i form) grzybów workowych i podstawkowych. Wśród znalezionych gatunków są grzyby bardzo rzadkie, znane z zaledwie jednego lub kilku stanowisk w Polsce (16 gatunków), a także wykazane w literaturze z Polski po raz pierwszy (5) oraz zagrożone i objęte ochroną (59), w tym Phellodon confluens - uznawany za wymarły.

Jak wskazano w części pierwszej artykułu (Ruszkiewicz-Michalska et al. 2012), badania przeprowadzone w BbPN są pierwszymi z planowanego przez Polskie Towarzystwo Mykologiczne cyklu inwentaryzacji w obiektach objętych różnymi formami ochrony. W wielu takich obiektach (parkach narodowych, rezerwatach, parkach krajobrazowych oraz obszarach Natura 2000) brak jest danych mykologicznych lub są fragmentaryczne albo wymagające aktualizacji. Podstawową przesłanką do prowadzenia badań inwentaryzacyjnych jest potrzeba uwzględniania grzybów w polityce ochrony przyrody, zarówno na obszarze parków narodowych, jak i w szerszej skali.
\end{abstract}

